\title{
Tetramethylpyrazine Analogue T-006 Exerts Neuroprotective Effects against 6-Hydroxydopamine-Induced Parkinson's Disease In Vitro and In Vivo
}

\author{
Hefeng Zhou, ${ }^{1,2}$ Min Shao, ${ }^{1}$ Xuanjun Yang, ${ }^{2,3}$ Chuwen Li $\left(\mathbb{D},{ }^{4}\right.$ Guozhen Cui ${ }^{1},{ }^{1}$ Cheng Gao, ${ }^{2}$ \\ Lijun Di, ${ }^{5}$ Hanbing Zhong $\oplus,{ }^{3}$ Yuqiang Wang $\oplus{ }^{6}{ }^{6}$ Zaijun Zhang $\oplus{ }^{6}{ }^{6}$ \\ and Simon Ming-Yuen Lee $\mathbb{D}^{2}$ \\ ${ }^{1}$ Department of Bioengineering, Zhuhai Campus of Zunyi Medical University, Zhuhai, China \\ ${ }^{2}$ State Key Laboratory of Quality Research in Chinese Medicine and Institute of Chinese Medical Sciences, University of Macau, \\ Macau, China \\ ${ }^{3}$ Department of Biology, South University of Science and Technology, Shenzhen, China \\ ${ }^{4}$ Key Laboratory of Molecular Target \& Clinical Pharmacology, School of Pharmaceutical Sciences, Guangzhou Medical University, \\ Guangzhou, China \\ ${ }^{5}$ Cancer Center, Faculty of Health Sciences, University of Macau, Macau, China \\ ${ }^{6}$ Institute of New Drug Research, College of Pharmacy, Jinan University, Guangzhou, China
}

Correspondence should be addressed to Zaijun Zhang; zaijunzhang@163.com and Simon Ming-Yuen Lee; simonlee@umac.mo

Received 14 March 2019; Revised 17 June 2019; Accepted 18 July 2019; Published 14 November 2019

Academic Editor: Rodrigo Franco

Copyright ( 2019 Hefeng Zhou et al. This is an open access article distributed under the Creative Commons Attribution License, which permits unrestricted use, distribution, and reproduction in any medium, provided the original work is properly cited.

Parkinson's disease (PD) is a neurodegenerative disorder characterized by the progressive loss of dopaminergic neurons in the substantia nigra pars compacta $(\mathrm{SNpc})$, and there is no cure for it at present. We have previously reported that the tetramethylpyrazine (TMP) derivative T-006 exhibited beneficial effects in Alzheimer's disease (AD) models. However, its effect on PD remains unclear. In the present study, we investigated the neuroprotective effects and underlying mechanisms of T-006 against 6-hydroxydopamine- (6-OHDA-) induced lesions in in vivo and in vitro PD models. Our results demonstrated that T-006 alleviated mitochondrial membrane potential loss and restored the energy metabolism and mitochondrial biogenesis that were induced by 6-OHDA in PC12 cells. In addition, animal experiments showed that administration of T-006 significantly attenuated the 6-OHDA-induced loss of tyrosine hydroxylase- (TH-) positive neurons in the SNpc, as well as dopaminergic nerve fibers in the striatum, and also increased the concentration of dopamine and its metabolites (DOPAC, HVA) in the striatum. Functional deficits were restored following T-006 treatment in 6-OHDA-lesioned mice, as demonstrated by improved motor coordination and rotational behavior. In addition, we found that the neuroprotective effects of T-006 were mediated, at least in part, by the activation of both the PKA/Akt/GSK-3 $\beta$ and CREB/PGC-1 $\alpha /$ NRF-1/TFAM pathways. In summary, our findings demonstrate that T-006 could be developed as a novel neuroprotective agent for PD, and the two pathways might be promising therapeutic targets for $\mathrm{PD}$.

\section{Introduction}

Parkinson's disease (PD) is the second most common neurodegenerative disorder worldwide, affecting up to $1 \%$ of the global aged population over 60 years [1]. It is mainly characterized by a progressive degeneration of dopaminergic neurons in the substantia nigra pars compacta $(\mathrm{SNpc})$ [2], which leads to striatal dopamine deficiency and results in motor symptoms such as postural instability, uncontrollable tremor, rigidity, and bradykinesia [3, 4]. Apart from motor symptoms, nonmotor symptoms, including autonomic dysfunction, sleep disturbances, cognitive problems, and depression, are commonly observed in PD [5]. Current treatments for $\mathrm{PD}$, including dopaminergic replacement therapy and 


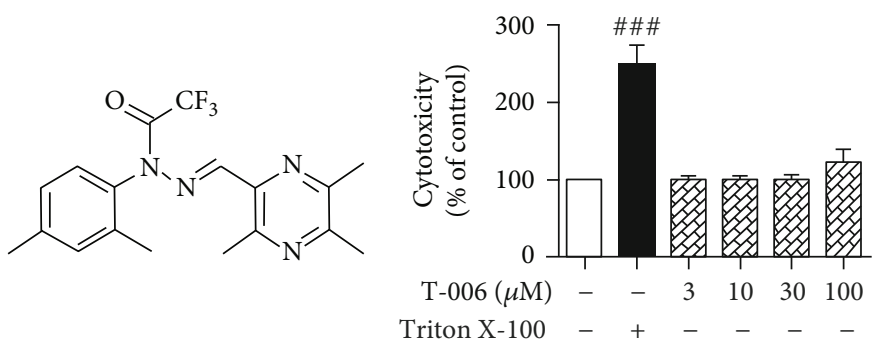

(a)

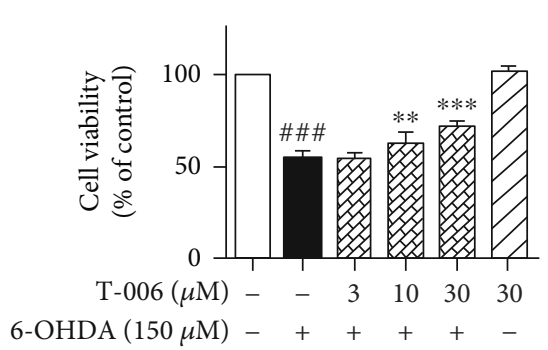

(c) (b)

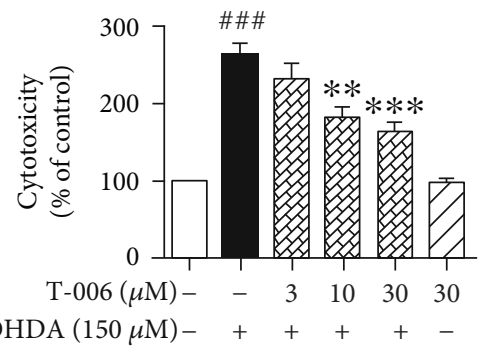

(d)

FIgure 1: Neuroprotective effect of T-006 on 6-OHDA-induced neurotoxicity in PC12 cells. (a) Chemical structure of T-006. (b) PC12 cells were treated with different concentrations of T-006 or Triton X-100 $(0.1 \%, v / v)$ for $24 \mathrm{~h}$, and then cytotoxicity was analyzed by an LDH kit. PC12 cells were pretreated with T-006 $(3,10$, and $30 \mu \mathrm{M})$ or $0.1 \%$ DMSO (vehicle control) for $12 \mathrm{~h}$ and then challenged with $150 \mu \mathrm{M} 6$ OHDA for an additional $24 \mathrm{~h}$. Cell viability (c) and cytotoxicity (d) were measured by MTT assay and LDH assay, respectively. Data from three independent experiments are represented as the mean $\pm \mathrm{SD}$. ${ }^{\# \# \#} P<0.001$ compared to the control group; ${ }^{* *} P<0.01$ and ${ }^{* * *} P<0.001$ compared to the 6-OHDA-treated group.

deep brain stimulation therapy, cannot halt or slow down the progression of the disease. With the aging of the world population, PD is considered an increasing burden on society and the world economy.

Although the etiology of PD has not been fully elucidated, accumulating evidence suggests that oxidative stress, mitochondrial dysfunction, and dysregulation of glucose play a significant role in the pathogenesis of PD [6-10]. The neurotoxin 6-hydroxydopamine (6-OHDA) is widely used to produce models of $\mathrm{PD}$. It has been shown that the toxicity of 6-OHDA corresponds to the increased generation of reactive oxygen species (ROS), deficient glycolytic activity, inhibition of the tricarboxylic acid (TCA) cycle, and direct inhibition of complex I and complex IV of the mitochondrial electron transport chain (ETC) $[11,12]$.

Glucose metabolism involves glycolysis and the pentose phosphate pathway (PPP) in the cytoplasm and the TCA cycle (Krebs cycle) and oxidative phosphorylation in mitochondria [13]. Glycolysis and the TCA cycle provide reducing equivalents for oxidative phosphorylation and finally generate ATP through the ETC in mitochondria. A decrease in glucose metabolism has been reported in PD [14]. In addition, dysfunction of the TCA cycle and oxidative phosphorylation leads to severe energy deficiency as well as increased generation of ROS in neurons $[10,15]$. Moreover, several signaling pathways regulate both glucose metabolism and mitochondrial biogenesis; for example, PI3K/Akt and PGC$1 \alpha /$ NRF- 1 activation was reported to exert a neuroprotective effect in PD [16-18].

We have reported that a multifunctional compound, $\mathrm{T}-006$, in which the methoxyphenyl group of J147 was replaced with tetramethylpyrazine (TMP) (see Figure 1(a)), exhibited beneficial effects in Alzheimer's disease (AD) models $[19,20]$. A previous study showed that T-006 could concurrently antagonize NMDA receptors and regulate the $\mathrm{PI} 3 \mathrm{~K} / \mathrm{Akt}$ pathway, thereby stimulating neurite outgrowth in PC12 cells and potentiating nerve growth factor- (NGF-) induced neuritogenesis [19]. T-006 could rescue iodoacetic acid-induced neuronal loss, prevent oxidative stressinduced neurotoxicity, and reduce glutamate-induced excitotoxicity in cerebellar granule neurons, as well as significantly ameliorate memory impairments in APP/PS1 transgenic mice [20]. However, its effect on the PD remains unclear. In the present study, we aimed to investigate the neuroprotective potential of T-006 and the underlying mechanisms of its action against 6-OHDA-induced neurotoxicity in in vivo and in vitro models.

\section{Materials and Methods}

2.1. Materials. 6-OHDA, dimethyl sulfoxide (DMSO), paraformaldehyde (PFA), and thiazolyl blue tetrazolium bromide (MTT) were purchased from Sigma-Aldrich (St. Louis, MO, USA). A lactate dehydrogenase (LDH) kit and cocktail were purchased from Roche Applied Science (Indianapolis, IN, USA). F-12K medium, FBS, HS, penicillin-streptomycin (PS), trypsin-EDTA, and PBS were purchased from Life Technologies (Grand Island, NY, USA). Enhanced chemiluminescence (ECL) solution was obtained from Thermo Fisher Scientific (Rockford, IL, USA). RIPA lysis buffer was bought from Beyotime Biotechnology (Shanghai, China). H-89 was purchased from Selleck Chemicals (Shanghai, 
China). SYBR ${ }^{\circledR}$ Premix Ex Taq $^{\mathrm{TM}}$ II kit was purchased from TaKaRa. (Dalian, China). Antibodies against p-PKA, PKA, p-Akt, Akt, p-CREB, CREB, p-PI3K, PI3K, p-GSK-3 $\beta$, GSK$3 \beta, \beta$-actin, lamin $\mathrm{B} 1$, and HRP-conjugated anti-rabbit IgG were purchased from Cell Signaling Technology (Boston, MA, USA). Antibodies against Nrf2, PGC-1 $\alpha$, NRF1, and TFAM were purchased from Santa Cruz Biotechnology (Santa Cruz, CA, USA). XF cell culture microplates and XF96 extracellular flux assay kits were purchased from Seahorse Bioscience (North Billerica, MA, USA). All other chemicals of analytical grade were purchased from local sources.

2.2. Cell Culture and Treatments. The rat adrenal rat pheochromocytoma PC12 cell line was purchased from the American Type Culture Collection (Manassas, VA, USA). Cells were cultured in F-12K medium supplemented with $10 \%(v / v)$ HS, 5\% $(v / v)$ FBS, and $1 \%(v / v)$ PS in a humidified atmosphere of $5 \% \mathrm{CO}_{2}$ and $95 \%$ air at $37^{\circ} \mathrm{C}$. Cells were treated with $\mathrm{T}-006$ when they reached about $70 \%$ confluence. A stock solution of T-006 was dissolved in DMSO and added directly to the culture media to a final concentration of $0.1 \%(v / v)$ DMSO. The control cells were treated with DMSO only.

2.3. Cell Viability and Cytotoxicity Analysis. The methods were described in detail elsewhere [21]. In brief, PC12 cells were seeded in 96-well plates $\left(8 \times 10^{3}\right.$ cells/well $)$ for $24 \mathrm{~h}$. After treatment with different concentrations of T-006 (ranging from 3 to $100 \mu \mathrm{M}$ ) for $24 \mathrm{~h}$, the supernatant was removed for LDH cytotoxicity analysis, while the remainder was used for MTT cell viability analysis. Absorbance was measured at $490 \mathrm{~nm}$ for $\mathrm{LDH}$ and $570 \mathrm{~nm}$ for MTT using a SpectraMax M5 (Wallace, Netherlands).

\subsection{Evaluation of Mitochondrial Membrane Potential $(\Delta \psi m)$.} JC-1 dye was used to monitor mitochondrial integrity [22]. Briefly, PC12 cells were seeded into black 96-well plates $\left(5 \times 10^{3}\right.$ cells/well $)$. At the end of treatment, the cells were incubated in the dark with $\mathrm{JC}-1(1 \mu \mathrm{g} / \mathrm{mL}$ in medium) at $37^{\circ} \mathrm{C}$ for $15 \mathrm{~min}$ and then washed twice with warm medium. The $\Delta \psi \mathrm{m}$ change was measured by flow cytometry (BD Biosciences, Franklin lakes, NJ, USA). The ratio of JC-1 red/green fluorescence intensity was calculated for the semiquantitative assessment of mitochondrial polarization states, and the value was normalized to the control group.

2.5. Preparation of Nuclear Extracts. Proteins in the nuclei of cells were isolated by the Nuclear and Cytoplasmic Protein Extraction Kit (Beyotime, Shanghai, China) according to the manufacturer's instructions. In brief, following treatments, PC12 cells were washed with ice-cold PBS, scraped in cold cytosol extraction buffer A with protease inhibitor cocktail, and incubated on ice for $10 \mathrm{~min}$, followed by the addition of cytoplasmic protein extraction agent $\mathrm{B}$. The mixture was centrifuged at $15,000 \mathrm{~g}$ for $5 \mathrm{~min}$ at $4^{\circ} \mathrm{C}$, the pelleted nuclei were lysed in a nuclear protein extraction agent supplemented with protease inhibitor cocktail and centrifuged at $12,000 \mathrm{~g}$ for $5 \mathrm{~min}$ at $4^{\circ} \mathrm{C}$, and the supernatant containing nuclear proteins was collected. The protein concentration of the nuclear was determined by the Bradford method.
PGC- $1 \alpha$ and NRF- 1 levels were determined by Western blot analysis as described below.

2.6. Western Blot Analysis. Protein levels were examined using Western blot analysis as previously described [23]. Briefly, after appropriate treatment, the collected cells were lysed with RIPA lysis buffer. For the brain samples, tissues were homogenized in RIPA lysis buffer supplemented with protease inhibitor PMSF and cocktail as per manufacturer's instruction to extract protein. Protein concentration was measured by a BCA protein assay kit. The same amounts of protein samples were electrophoresed on SDS-polyacrylamide gel, transferred to PVDF membrane. Membranes were subsequently incubated overnight at $4{ }^{\circ} \mathrm{C}$ with various primary antibodies in $5 \%$ fat-free dry milk-TBST [each antibody was diluted at 1:1000: phospho-PKA (Thr197), PKA, phosphoAkt (Ser473), Akt, phospho-CREB (Ser133), CREB, phospho-GSK-3 $\beta$ (Ser9), GSK-3 $\beta$, and lamin B1, except $\beta$-actin (1:2000), Nrf2 (1:500), PGC-1 $\alpha$ (1:500), NRF1 (1:500), and TFAM (1:500)]. The blots were then incubated with HRP-conjugated secondary antibody in TBST at a 1:5000 dilution for $1 \mathrm{~h}$ at room temperature. Protein bands were visualized with an enhanced chemiluminescence (ECL) kit. Blots were repeated at least three times for every condition. After development, the density of the bands was quantified by Image Lab Software (Bio-Rad, Hercules, CA, USA).

2.7. Analysis of mtDNA Copy Number. The copy number of mtDNA was determined by real-time quantitative PCR as previously described, with minor changes [24]. Real-time PCR with the SYBR ${ }^{\circledR}$ Premix Ex Taq ${ }^{\mathrm{TM}}$ II kit was performed on an Mx3005P qPCR system (Agilent Technologies, Santa Clara, CA, USA). The following primer sequences were used: D-loop-F, GGTTCTTACTTCAGGGCCATCA; Dloop-R, GATTAGACCCTGTACCATCGAGAT; 18s rRNAF GCAATTATTCCCCATGAACG; 18s rRNA-R, GGCCTC ACTAAACCATCCAA. Relative mtDNA copy number was calculated with the $2^{-\triangle \triangle \mathrm{Ct}}$ method.

2.8. LC-MS Analysis of Cellular Metabolites and Neurotransmitters in the Striatum. Intracellular metabolites in glycolysis and tricarboxylic acid cycle were analyzed by an Agilent 1200 series HPLC system coupled with an Applied Biosystems/MDS Sciex 4000 QTRAP mass spectrometer [25]. In brief, PC12 cells were seeded in 6-well plates $\left(5 \times 10^{5}\right.$ cells/well $)$. After treatment with T-006 $(30 \mu \mathrm{M})$ for $12 \mathrm{~h}$, cells were washed once with PBS and lysed in $80 \%$ (vol/vol) methanol at $-78^{\circ} \mathrm{C}$ to extract intracellular polar metabolites. The homogenate was mixed with $200 \mu \mathrm{L}$ of $\mathrm{ddH}_{2} \mathrm{O}$ and centrifuged at $15,000 \mathrm{~g}$ for $15 \mathrm{~min}$ at $4^{\circ} \mathrm{C}$. The supernatants were ultrafiltered using a $5 \mathrm{kDa}$ ultrafiltration membrane to remove the proteins. The filtrate was lyophilized, dissolved in $50 \mu \mathrm{L}$ of $\mathrm{ddH}_{2} \mathrm{O}$, and analyzed using LC-MS.

After the evaluation of animal behavioral tests, mice (15 mice per group) were killed by exposure to carbon dioxide and striata were then quickly dissected on ice. Each striatum was weighed and homogenized in $100 \mu \mathrm{L} \mathrm{ddH}_{2} \mathrm{O}$. Lysates were centrifuged at $15,000 \mathrm{~g}$ for $15 \mathrm{~min}$ at $4^{\circ} \mathrm{C}$. The supernatant $(80 \mu \mathrm{L})$ was added with $160 \mu \mathrm{L}$ acetonitrile, vortex, 
centrifuged with $15000 \mathrm{rpm}$ for $5 \mathrm{~min}$ at $4^{\circ} \mathrm{C}$. Around $200 \mu \mathrm{L}$ supernatant was collected for further LC-MS analysis [26].

In dopamine LC-MS analysis, $100 \mathrm{ppb}$ of 3,4-dihydroxybenzylamine hydrobromide (DHBA) was spiked into each sample as the internal standard. $10 \mu \mathrm{L}$ of the sample was injected onto a reversed-phase column (Synergi ${ }^{\mathrm{TM}} 4 \mu \mathrm{m}$ Polar-RP $80 \AA, 150 \times 4.6 \mathrm{~mm}$ ) with an Agilent 1200 series HPLC system. The sample injected was analyzed with a gradient of mobile phase consisting of $0.1 \%$ formic acid in water (solvent A) and acetonitrile (solvent B) in $200 \mu \mathrm{L} / \mathrm{min}$, respectively. The details of the gradient profile were as follows: $0-10 \mathrm{~min}, 50-100 \% \mathrm{~B} ; 10-15 \mathrm{~min}, 100 \% \mathrm{~B} ; 15-15.1 \mathrm{~min}$, $100-50 \% \mathrm{~B}$; and $15.1-30 \mathrm{~min}, 50 \% \mathrm{~B}$. The LC-MS data is collected by a QTRAP 4000 system operating positive MRM mode. The ion source parameters were as follows: capillary voltage $5500 \mathrm{~V}$; source temperature $150^{\circ} \mathrm{C}$; curtain gas 20 psi; GS1 30 psi; and GS2 30 psi.

In the LC-MS analysis for homovanillic acid (HVA) and dihyrophenylacetic acid (DHPA), 1 ppb of acephate (ACE) was spiked into each sample as the internal standard. The gradient profile used in the analysis was similar to that in the dopamine analysis, except solvents $\mathrm{A}$ and $\mathrm{B}$ which were replaced with water (solvent $\mathrm{C}$ ) and acetonitrile (solvent D), respectively. The LC-MS data is collected by the same mass spectrometer system operating in negative MRM mode. The ion source parameters were similar as in the dopamine analysis, except the capillary voltage which became $-4500 \mathrm{~V}$.

\subsection{Oxygen Consumption Rate (OCR) and Extracellular} Acidification Rate (ECAR) Measurements. OCR and ECAR were measured with the XF extracellular flux analyzer (Seahorse Bioscience) as previously described [27]. In brief, PC12 cells $\left(2 \times 10^{4}\right.$ cells/well $)$ were seeded into Seahorse XF24 microplates and incubated in a humidified $5 \% \mathrm{CO}_{2}$ atmosphere for $24 \mathrm{~h}$. On the day of the experiment, the medium was replaced with Seahorse base medium and incubated in a $\mathrm{CO}_{2}$-free $37^{\circ} \mathrm{C}$ incubator for $1 \mathrm{~h}$. For measuring OCR, $1 \mu \mathrm{M}$ oligomycin A, $1 \mu \mathrm{M}$ carbonyl cyanide-4-(trifluoromethoxy) phenylhydrazone (FCCP), and $1 \mu \mathrm{M}$ rotenone $+1 \mu \mathrm{M}$ antimycin A were loading sequentially whereas, for ECAR, $25 \mathrm{mM}$ glucose, $1 \mu \mathrm{M}$ oligomycin $\mathrm{A}$, and $100 \mu \mathrm{M} 2$-DG were applied. After finishing the XF assay, the cells were lysed with RIPA buffer $(200 \mu \mathrm{L} /$ well $)$ and the protein concentration was measured by BCA assay. The OCR and ECAR rates were normalized to the protein content and presented as pmols $/ \mathrm{min} / \mathrm{mg}$ protein and $\mathrm{mpH} / \mathrm{min} / \mathrm{mg}$ protein, respectively.

2.10. Animals and Treatment. Eight-week-old male C57BL/6 mice (purchased from the Medical Experimental Animal Center of Guangdong, China) were housed in standard cages with free access to food and water at $25^{\circ} \mathrm{C}$ following a $12 \mathrm{~h}: 12 \mathrm{~h}$ light/dark cycle. All animal experiments were approved by the Research Ethics Committee of Institute of Chinese Medical Sciences, University of Macau.

Animals were randomly divided into 4 groups (15 mice per group): control group, sham group (injected with saline solution and oral gavage with olive oil), lesion group (injected with 6-OHDA and gavaged with olive oil), and
T-006-treated lesion group (injected with 6-OHDA and gavaged with T-006).

The surgery was mostly based on unilateral stereotaxic injection of 6-OHDA. Mice were deeply anesthetized by $1 \%$ pentobarbital sodium $(50 \mathrm{mg} / \mathrm{kg})$ and placed in a stereotaxic frame. A total amount of $10 \mu \mathrm{g} 6-\mathrm{OHDA}(5 \mu \mathrm{g} / \mu \mathrm{L}$, dissolved in $0.9 \% \mathrm{NaCl} / 0.02 \%$ ascorbic acid) was stereotaxically injected into the right $\mathrm{SNpc}$ [28] (coordinates relative to $\mathrm{AP},-3.1 \mathrm{~mm}$; ML, $+1.2 \mathrm{~mm}$; and DV, $-4.2 \mathrm{~mm}$ from the skull) at an injection rate of $0.5 \mu \mathrm{L} / \mathrm{min}$. After injection, the needle was left in place for $5 \mathrm{~min}$ before removal. The sham group was stereotaxically injected with the same volume $(2 \mu \mathrm{L})$ of $0.2 \% \mathrm{~L}$-ascorbic acid/saline only.

The experimental timeline is shown in Figure 2(a). For the sham group and lesion group, mice received olive oil $(0.2 \mathrm{ml} /$ day $)$ by oral gavage once daily. Mice in the T-006treated lesion group received T-006 $(3 \mathrm{mg} / \mathrm{kg} /$ day, dissolved in olive oil) by oral gavage once daily, which begin 2 days prior to 6-OHDA lesioning and continued for another 14 days. This dose of T-006 (3 mg/kg/day) was selected on the basis of previously published reports [20].

2.11. Behavioral Analysis. For the rotation test, mice received a subcutaneous injection of apomorphine $(0.5 \mathrm{mg} / \mathrm{kg})$ in $0.9 \%$ saline [26]. Turning behavior was monitored directly after injection for $15 \mathrm{~min}$ by videotaping. Each $360^{\circ}$ turn of the body axis was manually counted as a rotation. Values were expressed as the mean of contralateral turns collected during the 5-15 min period.

For the rotarod test, the mice were trained five days to reach a stable performance, and five mice separated by large black disks were tested simultaneously. After the mice had been placed on the rotarod at a constant rotation speed of $22 \mathrm{rpm}[26,29]$, the trial was started and sustained for $10 \mathrm{~min}$. The trial stopped when the mouse fell down (activating a switch that automatically stopped the timer) or when 10 min had elapsed.

2.12. Immunohistochemistry. Mice were transcardially perfused with PFA in PBS (pH 7.4). Brains were dissected, post-fixed in PFA overnight, and then dehydrated in 30\% sucrose in PBS at $4^{\circ} \mathrm{C}$. Sections were incubated in a monoclonal anti-TH primary antibody $(1: 400$, Millipore; MAB318) for $48 \mathrm{~h}$ at $4^{\circ} \mathrm{C}$, washed in PBS, and incubated with secondary anti-rabbit-HRP antibody. Immunostaining was visualized after 3-3' diaminobenzidine (DAB) staining (Vector Laboratories, Burlingame, CA, USA) using bright field microscopy (Leica, Wetzlar, Germany). Five sections through the SNc areas $(-5.3 \mathrm{~mm}$ AP from Bregma) were randomly selected from each animal. The unbiased stereological counting of $\mathrm{TH}$-positive neurons was performed bilaterally in the SNc areas. A detailed description of the stereological counting procedure is provided in supplementary materials (S1 File). The number of TH-positive neurons in the ipsilateral side and contralateral control side was calculated. Values were then expressed as \% of the contralateral control side. For quantitative assessment of $\mathrm{TH}$-immunoreactive fiber density in the striatum region, the optical density was measured by ImageJ software. Briefly, the background was firstly removed 


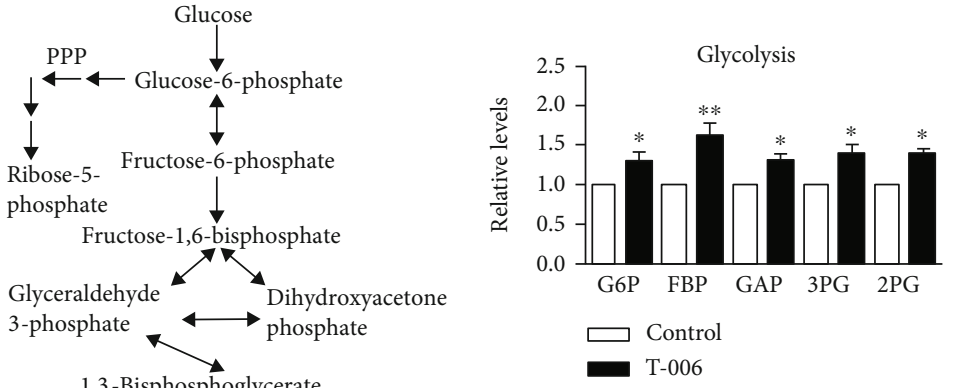

(b)

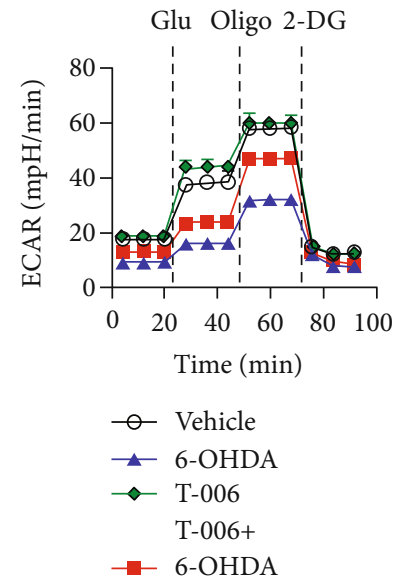

(e)

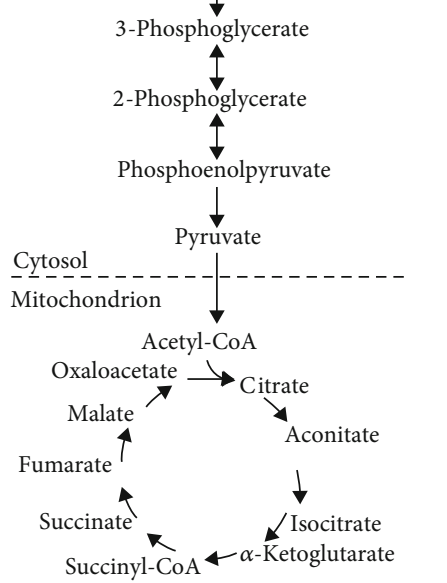

(a)

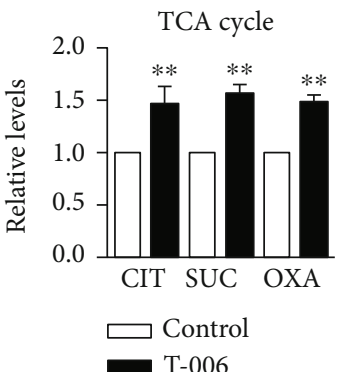

(c)

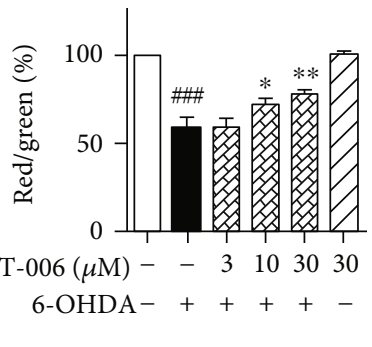

(d)

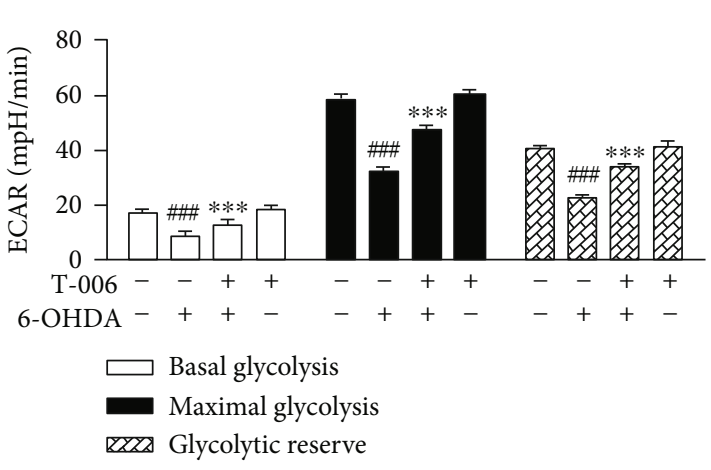

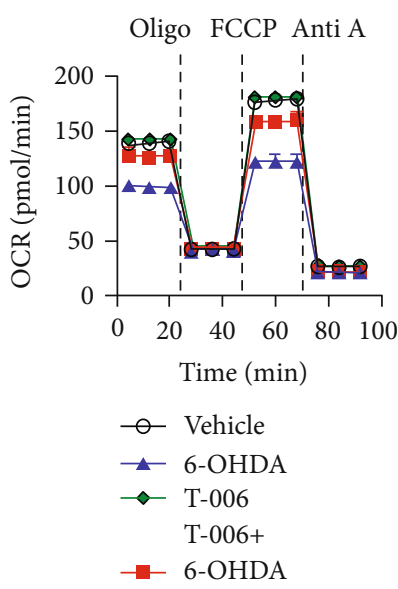

(g)

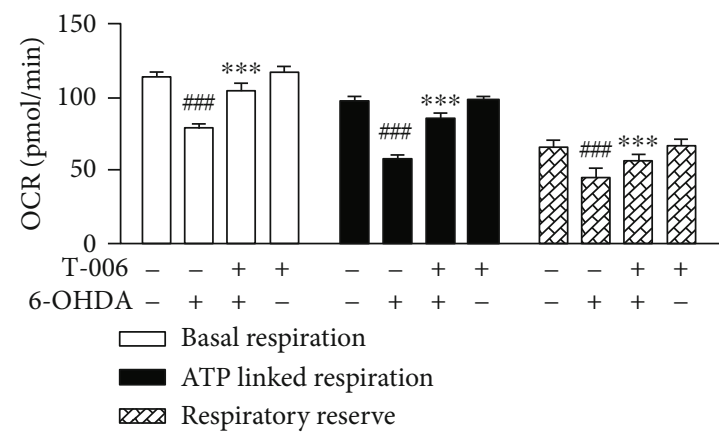

(h)

FIgURE 2: T-006 increases energy metabolism and mitochondrial functions in PC12 cells. PC12 cells were pretreated with T-006 (30 $\mu \mathrm{M})$ or $0.1 \%$ DMSO (vehicle control) for $12 \mathrm{~h}$; intracellular glycolysis and TCA cycle metabolites were measured by LC-MS. (a) A simplified metabolic flow diagram of glycolysis and the TCA cycle (red, upregulated). (b) Relative levels of enhanced glycolytic intermediates. (c) Relative levels of enhanced TCA cycle intermediates. G6P: glucose 6-phosphate; FBP: fructose 1,6-phosphate; GAP: glyceraldehyde 3phosphate; 3PG: 3-phosphoglycerate; 2PG: 2-phosphoglycerate; CIT: citrate; SUC: succinate; OXA: oxaloacetate. PC12 cells were pretreated with T-006 $(30 \mu \mathrm{M})$ or $0.1 \%$ DMSO for $12 \mathrm{~h}$ and then challenged with or without $150 \mu \mathrm{M} 6$-OHDA for another $12 \mathrm{~h}$. (d) PC12 cells were pretreated with T-006 $(3,10$, and $30 \mu \mathrm{M})$ or $0.1 \%$ DMSO for $12 \mathrm{~h}$ and then challenged with or without $150 \mu \mathrm{M} 6-\mathrm{OHDA}$ for an additional $6 \mathrm{~h} . \Delta \psi \mathrm{m}$ was determined by flow cytometry. (e) The extracellular acidification rate (ECAR) was measured as an indicator of glycolytic activity using an XF24 Extracellular Flux Analyzer (normalized to protein content). The sequential injection of glucose, oligomycin, and 2-DG is indicated by vertical lines. (f) Quantitative data of basal glycolysis, maximal glycolysis, and glycolytic reserve (data from (e)). (g) The oxygen consumption rate (OCR) was measured as the mitochondrial OXPHOS activity. The sequential injection of mitochondrial inhibitors is indicated by vertical lines. (h) Quantitative data of basal respiration, ATP-linked respiration, and respiratory reserve (data from $(\mathrm{g})$ ). Data from three independent experiments are represented as the mean $\pm \mathrm{SD}$. \#\# $P<0.001$ as compared to the control group; ${ }^{*} P<0.05,{ }^{* *} P<0.01$, and ${ }^{* * *} P<0.001$, as compared to the 6-OHDA-treated group. 
by subtracting values obtained from regions where no staining was observed. Values were expressed as \% of the optical density of the ipsilateral side compared with that of the contralateral control side.

2.13. Statistical Analysis. Statistical analyses were performed with one-way ANOVA followed by Tukey's multiple comparison test (two or more groups) and Student's $t$-test (two groups) using GraphPad Prism 6.0 software (GraphPad Software Inc., San Diego, CA, USA). All experiments were performed three times and in triplicate or quadruplicate. Data are presented as the means \pm SD. Significance was defined as $P<0.05$.

\section{Results}

3.1. T-006 Attenuates 6-OHDA-Induced Neurotoxicity in PC12 Cells. Firstly, we investigated the cytotoxicity of T006: PC12 cells were treated with different concentrations of T-006 for $24 \mathrm{~h}$, and cell viability was subsequently determined by MTT assay. As shown in Figure 1(b), T-006, at up to $30 \mu \mathrm{M}$, did not have any cytotoxic effects on PC12 cells, and $30 \mu \mathrm{M}$ T-006 were used in further experiments. To test the protective effects of T-006 against 6-OHDA-induced neurotoxicity, PC12 cells were pretreated with T-006 for $12 \mathrm{~h}$ before exposure to $150 \mu \mathrm{M}$ 6-OHDA for $24 \mathrm{~h}$. The results of MTT assay showed that the viability of cells incubated with $150 \mu \mathrm{M}$ 6-OHDA for $24 \mathrm{~h}$ was $54.2 \%$ (Figure 1(c)), whereas pretreatment with 10 and $30 \mu \mathrm{M}$ of T-006 significantly increased cell viability to $62.6 \%$ and $71.3 \%$, respectively, compared with the control group (100\%). In addition, the neuroprotective activity of T-006 was confirmed by LDH assay. As shown in Figure 1(d), pretreatment with T-006 at 10 and $30 \mu \mathrm{M}$ for $12 \mathrm{~h}$ significantly decreased 6-OHDA-induced $\mathrm{LDH}$ leakage from $264.2 \%$ to $183.3 \%$ and $165.1 \%$, respectively.

\subsection{T-006 Increases Energy Metabolism and Mitochondrial} Functions in PC12 Cells. The toxicity of 6-OHDA was associated with the collapse of cellular bioenergetics [30, 31]. We first defined the metabolic profiles of PC12 cells by measuring glycolytic and TCA cycle intermediates using LC-MS (Figure 2(a)). The relative levels of elevated intermediates were quantified, as shown in Figure 2(b). T-006 treatment resulted in an increase in the glycolytic pathway metabolites glucose 6-phosphate, fructose 6-phosphate, glyceraldehyde 3-phosphate, 3-phosphoglycerate, and 2-phosphoglycerate. T-006 also induced an increase in metabolites associated with the TCA cycle pathway, as evidenced by an increase in citrate, succinate, and oxaloacetate (Figure 2(c)).

To further characterize the involvement of T-006 on 6OHDA-induced glycolysis dysfunction, we measured the ECAR under glucose starvation conditions and subsequent addition of oligomycin (Oligo) and 2-deoxyglucose (2-DG) was done in special 24-well plates equipped with oxygen sensors (Figure 2(e)). The ECAR was recorded in a glucose-free medium as basal ECAR; subsequently, the ECAR increase following the addition of glucose (Glu) can be sued to estab- lish the glycolysis rate. Oligo (ATP synthase inhibitor) was used to stimulate maximal ECAR, effectively shifting metabolism from oxidative phosphorylation to glycolysis. The final addition of the glycolysis inhibitor 2-DG abolished the overall glycolysis. The difference between maximal and basal ECAR is considered the glycolytic reserve capacity of cells. PC12 cells treated with 6-OHDA for $12 \mathrm{~h}$ had lower basal glycolysis, maximal glycolysis, and glycolytic reserve capacity (Figure 2(f)) compared to the control, suggesting that 6OHDA inhibits glycolysis activity. Pretreatments with $30 \mu \mathrm{M}$ T-006 significantly alleviate 6-OHDA-induced glycolytic rate collapse, as measured by ECAR, indicating that $\mathrm{T}$ 006 enhances glycolysis.

6-OHDA has also been shown to impair oxidative phosphorylation through direct inhibition of the activity of mitochondrial complexes I and IV [31-33]. To study whether T006 prevents 6-OHDA-induced loss of mitochondrial respiratory capacity, we further assess mitochondrial function by the measuring live cell OCR. As shown in Figure 2(g), the OCR profiles, in the basal state and after the addition of oligo, FCCP, and rotenone plus antimycin A (Anti A), were determined in real time. Following the injection of oligo, basal OCR is expected to drop and the extent of the drop can be used to deduce ATP production (ATP linked respiration). Next, cells were exposed to FCCP, which is an uncoupler of mitochondrial oxidative phosphorylation that dissipates the transport of electrons from ATP production, resulting in maximal respiration. The difference between basal and maximal OCR is the reserve capacity. Finally, inhibitors of complex I and III were added to shut down the electron transfer, allowing the calculation of the nonmitochondrial respiration. 6-OHDA caused a decrease in basal respiration, ATP linked respiration, and respiratory reserve capacity (Figure 2(h)) compared to the control. T-006 prevented the mitochondrial OCR impairments induced by 6 OHDA and by itself also improved the basal OCR, ATPlinked OCR, and reserve capacity. This result revealed that most of the 6-OHDA-induced impairment in glycolysis and oxidative phosphorylation was abolished by T-006 pretreatment. Finally, mitochondrial function were measured using JC-1 staining. As indicated in Figure 2(d), treatment with 6-OHDA significantly decreased intracellular $\Delta \psi \mathrm{m}$ to $58.8 \%$ of the control cell value, while pretreatment with T-006 markedly restored 6-OHDA-mediated intracellular $\Delta \psi \mathrm{m}$ to $77.9 \%$ of the control value.

Taken together, these findings suggest that T-006 could protect against 6-OHDA-induced neurotoxicity partly via increasing glycolysis and mitochondrial respiratory function in PC12 cells.

\subsection{T-006 Increases Glycolysis, TCA Cycle, and Mitochondrial} Respiration through the PKA/Akt/GSK-3 $\beta$ Signaling Pathway. Next, we investigated the underlying molecular mechanism by which T-006 increased glycolysis, TCA cycle, and mitochondrial respiration. Activation of PI3K/Akt/GSK$3 \beta$ signaling is known to result in enhanced glycolysis [34-37] and mitochondrial respiration [38, 39]. The effects of T-006 on this signaling pathway were studied by Western blot analysis. As shown in Figures 3(a) and 3(b), 

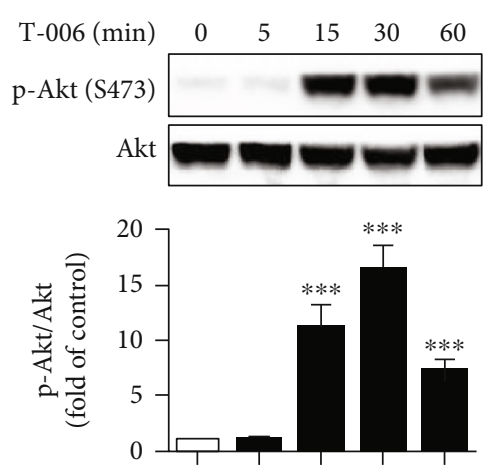

(a)

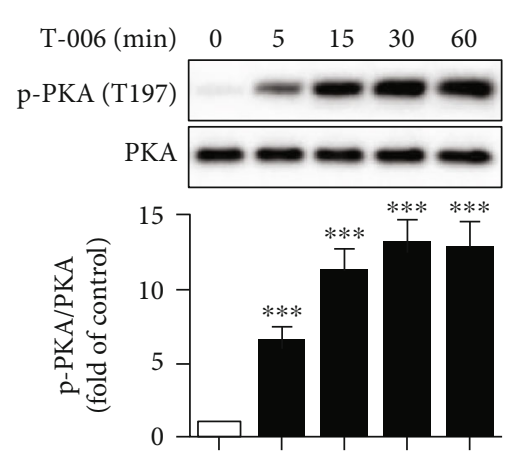

(c)

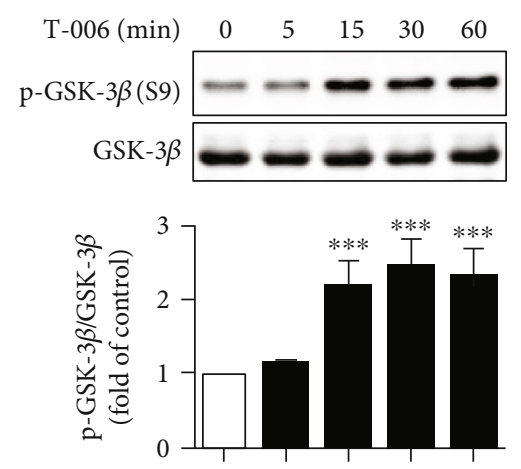

(b)

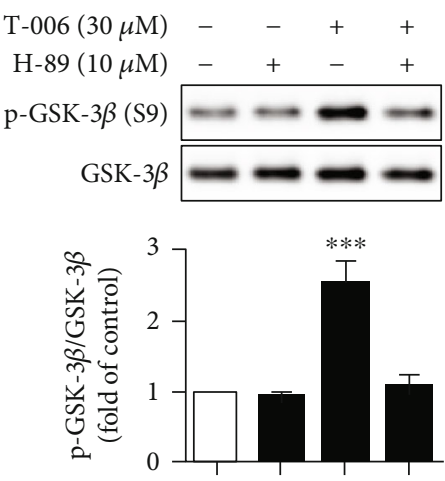

(d)
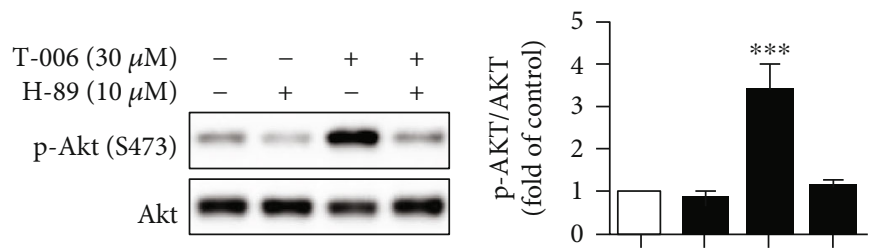

(e)

FIGURE 3: T-006 increases glycolysis, the TCA cycle, and mitochondrial respiration through the PKA/Akt/GSK-3 $\beta$ signaling pathway. PC12 cells were treated with T-006 $(30 \mu \mathrm{M})$ as indicated, and the expression ratios of phosphorylated Akt/total Akt (a), p-GSK-3 $\beta /$ total GSK-3 $\beta$ (b), and p-PKA/total PKA (c) were detected by Western blot analysis. Cells were pretreated with or without PKA inhibitor H-89 (10 $\mu \mathrm{M})$ for $1 \mathrm{~h}$ before exposure to T-006 $(30 \mu \mathrm{M})$ for $30 \mathrm{~min}$. The expression ratios of p-GSK-3 $\beta /$ total GSK-3 $\beta$ (d) and p-Akt/total Akt (e) were detected by Western blot analysis (densitometry readings are shown at the bottom of the blots). Representative Western blot and data analysis of three independent experiments are shown. Data are represented as the means $\pm \mathrm{SD}$. ${ }^{* * *} P<0.001$ compared to control.

treatment with T-006 apparently increased the phosphorylation of Akt at Ser473 (active) and GSK-3 $\beta$ at Ser9 (inactive), without affecting the total level of Akt and GSK-3 $\beta$, respectively. However, T-006 did not change the phosphorylation of PI3K (data not shown). Several groups reported that Akt could be activated by PKA [40, 41]; we found that T-006 significantly induced the phosphorylation of PKA (Figure 3(c)). To further examine whether T-006 activates Akt/GSK-3 $\beta$ through PKA, H-89 (a PKA inhibitor) was used to pretreat PC12 cells prior to T-006 stimulation. As predicted, pretreatment with $\mathrm{H}-89$ reversed T-006's effects on the phosphorylation of GSK-3 $\beta$ (Figure 3(d)) and Akt (Figure 3(e)). The results indicate that Akt activation and GSK-3 $\beta$ inactivation are regulated by PKA, and T-006 enhances glycolysis, TCA cycle, and mitochondrial respiration in $\mathrm{PC12}$ cells through the PKA/Akt/GSK-3 $\beta$ signaling pathway.
3.4. T-006 Protects PC12 Cells against 6-OHDA-Induced Neurotoxicity through Enhancing Mitochondrial Biogenesis via the PKA/CREB/PGC-1 $\alpha$ Pathway. We evaluated the effects of T-006 on mitochondrial biogenesis by investigating the expression of these three important transcriptional factors. As shown in Figure 4(a), PC12 cells were treated with T-006 for $24 \mathrm{~h}$, resulting in a significant increase in the nuclear content of PGC- $1 \alpha$ and its downstream target nuclear respiratory factor 1 (NRF-1). Furthermore, it was observed that the expression level of TFAM also increased significantly under these conditions (Figure 4(b)). To verify whether the elevation of nuclear PGC-1 $\alpha, \mathrm{NRF} 1$, and TFAM increases mitochondrial biogenesis, we investigated the mitochondrial DNA (mtDNA) content by real-time PCR. The results indicated that the mtDNA copy number was increased approximately 2 -fold after $24 \mathrm{~h}$ exposure of PC12 cells 

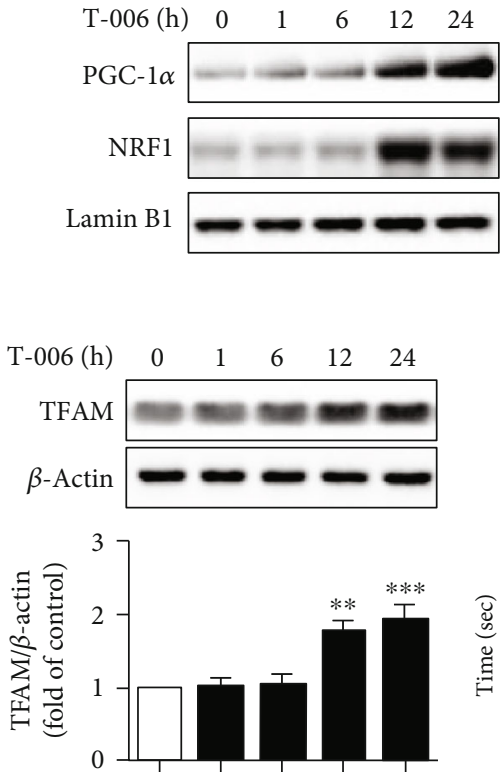

(b)

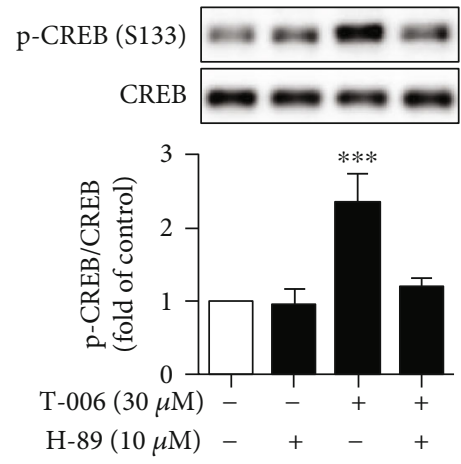

(e)

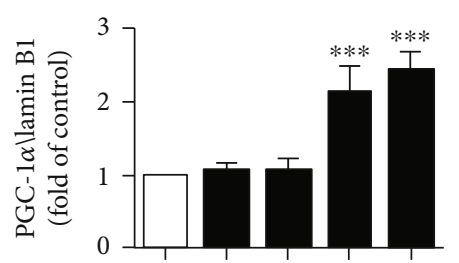

(a)

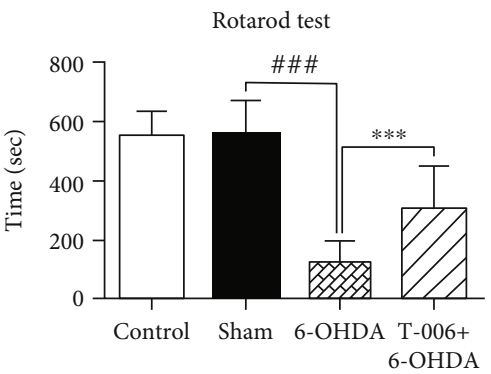

(c)

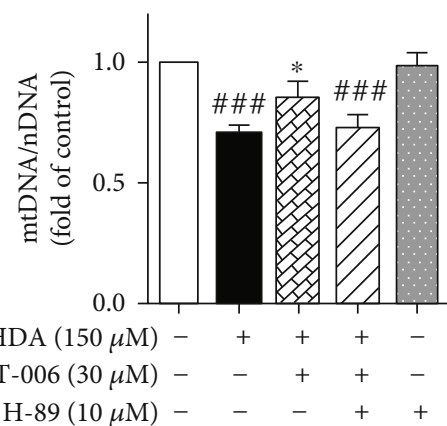

(f)
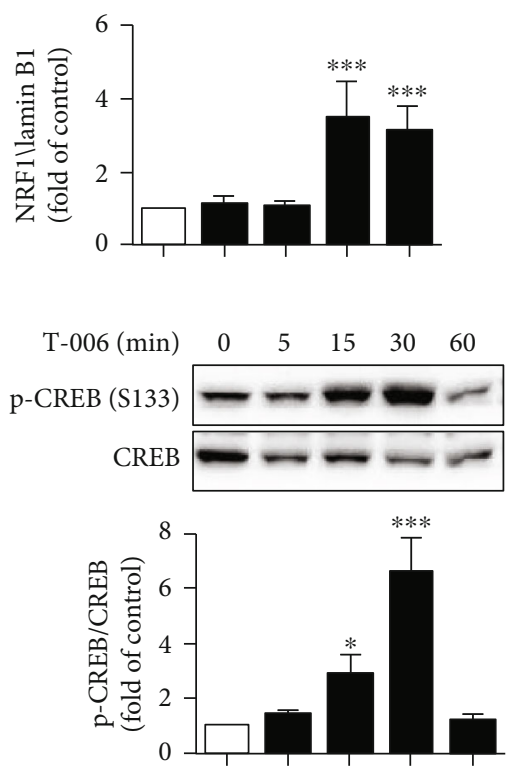

(d)

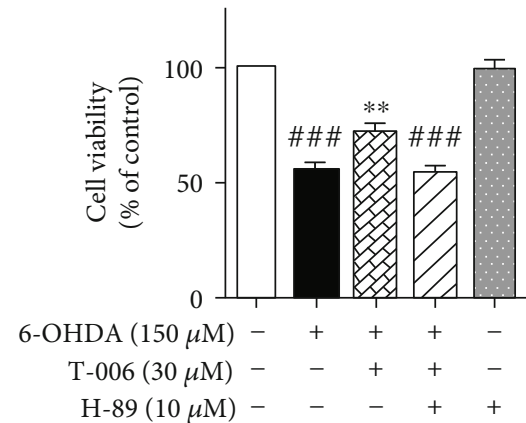

(g)

FIGURE 4: Effects of T-006 on the PKA/CREB/NRF1 signaling pathway and mitochondrial biogenesis in PC12 cells. PC12 cells were treated with T-006 $(30 \mu \mathrm{M})$ at the indicated time points, and the expression of nuclear PGC-1 $\alpha$ and NRF1 (a) and cytoplasmic TFAM (b) were detected by Western blot. (c) Cells were incubated with T-006 $(30 \mu \mathrm{M})$ as indicated, and the mtDNA content was quantified by quantitative real-time PCR. (d) The cells were treated with T-006 and harvested at the indicated times; the expression of phosphorylated CREB/total CREB was detected by Western blot analysis. (e) Cells were pretreated with or without PKA inhibitor H-89 (10 $\mu \mathrm{M})$ for $1 \mathrm{~h}$ and then treated with T-006 $(30 \mu \mathrm{M})$ for $30 \mathrm{~min}$. The expression ratios of phosphorylated CREB/total CREB were detected by Western blot. Representative Western blot and data analysis of three independent experiments are shown. Data are represented as the means \pm SD. ${ }^{*} P<0.05,{ }^{* *} P<0.01$, and ${ }^{* * *} P<0.001$, as compared to control. PC12 cells were pretreated with T-006 (30 $\left.\mu \mathrm{M}\right)$ for $12 \mathrm{~h}$ before treatment with H-89 $(10 \mu \mathrm{M})$ for $1 \mathrm{~h}$ and then challenged with $150 \mu \mathrm{M}$ 6-OHDA for an additional $24 \mathrm{~h}$; mtDNA content was quantified by quantitative real-time PCR (f). Cell viability was measured by MTT assay (g). Data from three independent experiments are represented as the mean \pm SD. ${ }^{\# \#} P<0.001$ compared to the control group; ${ }^{* *} P<0.01$ compared to the 6-OHDA-treated group.

to T-006 (Figure 4(c)). Furthermore, treatment with T006 for $15 \mathrm{~min}$ was sufficient to enhance the phosphorylation of CREB; however, total CREB levels remained consistent (Figure 4(d)). To test whether alterations in PKA activity explained the increased CREB phosphorylation upon T-006 treatment, the cells were preincubated with $10 \mu \mathrm{M}$ PKA inhibitor $\mathrm{H}-89$ for $1 \mathrm{~h}$ before T-006 addition, and it was shown that $\mathrm{H}-89$ completely abolished the T-006-induced phosphorylation of CREB (Figure 4(e)). We also found that inhibition of PKA by $\mathrm{H}-89$ blocked the increase in the mtDNA copy number (Figure 4(f)). To further confirm whether T-006 protects against 6-OHDAinduced cytotoxicity through enhancing mitochondrial biogenesis, PC12 cells were pretreated with $10 \mu \mathrm{M} \mathrm{H}-89$ for $1 \mathrm{~h}$ before T-006 addition; the results of MTT assay indicated that the protective effect of T-006 was abolished by H-89 (Figure 4(g)). Taken together, these data demonstrate that T-006 protects PC12 cells against 6-OHDA-induced neurotoxicity through enhancing mitochondrial biogenesis via the PKA/CREB/PGC- $1 \alpha$ pathway. 


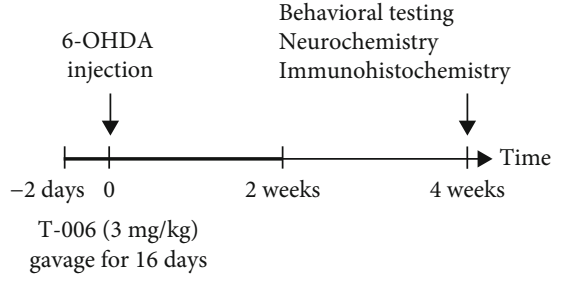

(a)

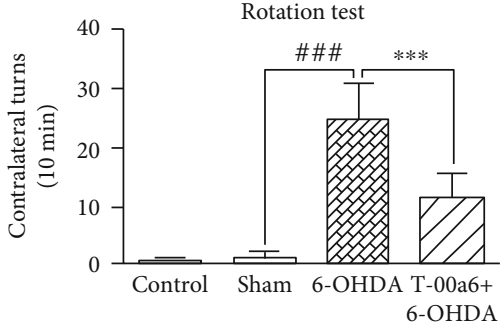

(b)
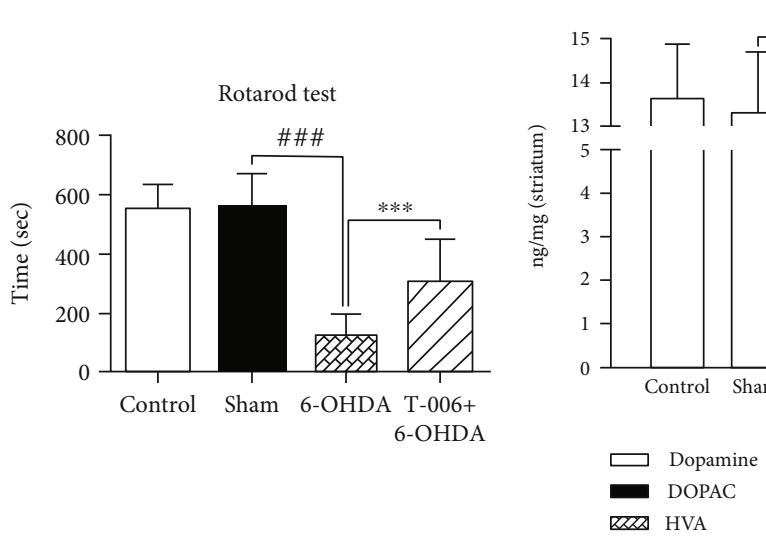

(c)

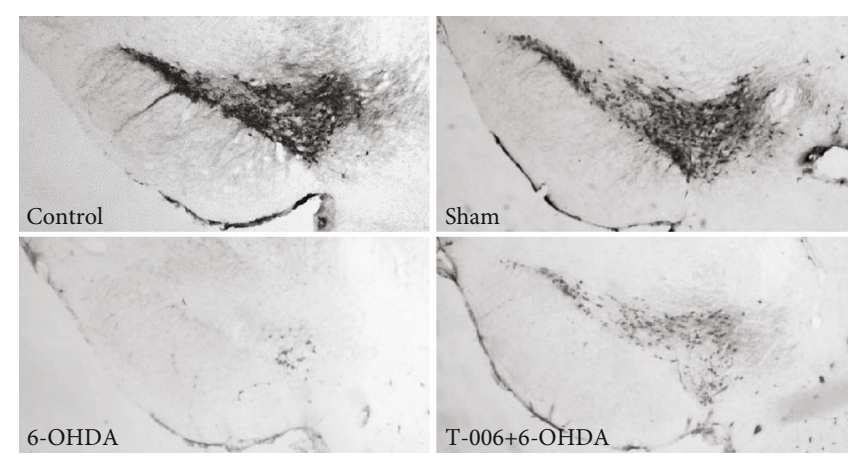

(e)
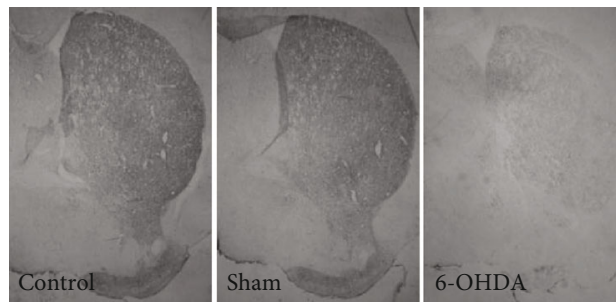

(g)

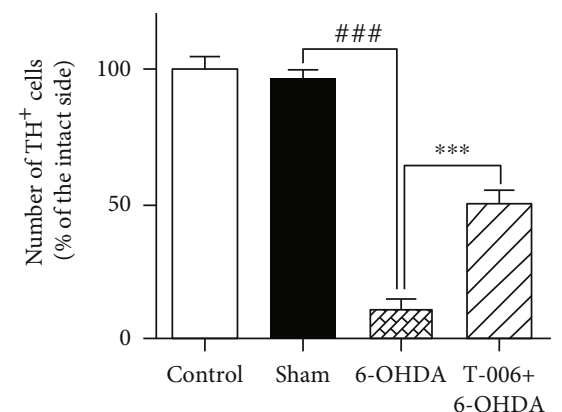

(f)

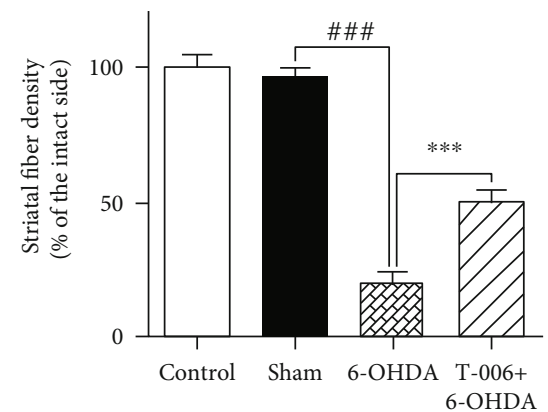

(h)

FIGURE 5: T-006 protects mice from 6-OHDA-induced neurotoxicity. (a) Experimental scheme. (b) Number of apomorphine-induced contralateral rotations (per $10 \mathrm{~min}$ ) in mice treated with T-006 (15 mice per group). (c) For the rotarod test, the time spent on the rotating drum before falling was recorded and analyzed for each mouse (15 mice per group). (d) Concentrations of dopamine and its major metabolites (DOPAC, HVA) in the striatum were determined by LC-MS (7 mice per group). (e) Representative images of TH immunostaining in the SNpc. (f) Quantification of TH-positive cells in the SNpc (8 mice per group). (g) Representative images of TH immunostaining in the striatum. (h) Optical density of TH-positive striatal fibers ( 8 mice per group). Data are represented as means \pm SD. ${ }^{\# \#} P<0.01$ and ${ }^{\# \#} P<0.001$ compared to the sham group; ${ }^{*} P<0.05,{ }^{* *} P<0.01$, and ${ }^{* * *} P<0.001$ compared to the 6-OHDA-treated group. 

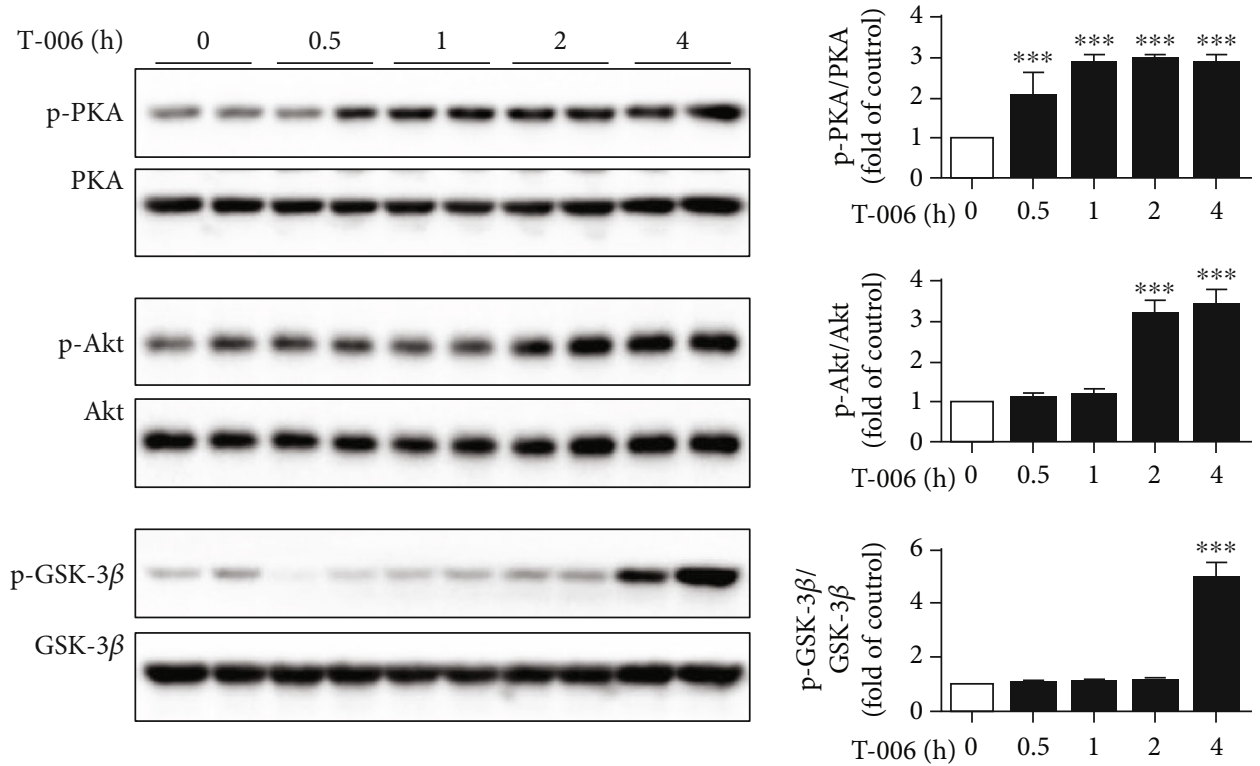

FIGURE 6: T-006 treatment activates PKA/Akt/GSK-3 $\beta$ signaling in brain tissues. C57BL/6 mice were dosed orally with either olive oil (for the $0 \mathrm{~h}$ time point control) or $3 \mathrm{mg} / \mathrm{kg}$ T-006 for $0.5,1,2$, and $4 \mathrm{~h}$ ( 2 mice per group). Whole brain tissues were harvested, and the homogenates were subjected to Western blot and semiquantified by densitometry. Representative immunoblots of total PKA, Akt, GSK-3 $\beta$, and their phosphorylated forms. Data are represented as means \pm SD. ${ }^{* * *} P<0.001$ compared to control.

3.5. T-006 Protects Mice from 6-OHDA-Induced Neurotoxicity. Encouraged by the cell-based results, we undertook studies to investigate whether T-006 protects mice from 6-OHDAinduced neurotoxicity in vivo. C57BL/6 mice were dosed orally with olive oil $(0.2 \mathrm{ml} /$ day $)$ or T-006 (3 mg/kg/day) once daily. Saline (vehicle) or 6-OHDA was stereotaxically injected into the right $\mathrm{SNpc}$ of eight-week-old mice at day 3. The mice were treated with olive oil or T-006 for a further 2 weeks (Figure 5(a)). The results of the apomorphine-induced rotation test are shown in Figure 5(b). We observed that PD model group (6-OHDA) mice presented with a high number of apomorphine-induced contralateral rotations compared with sham group (saline-injected) mice. This behavior was significantly attenuated by treatment with T-006 in lesioned mice. For the rotarod test, the mice were trained five days to reach a stable performance; then, the time for which each mouse stayed on the rotating drum $(22 \mathrm{rpm} / \mathrm{min})$ before falling was recorded and analyzed. As shown in Figure 5(c), the 6OHDA group exhibited a significant decrease in the time spent on the rotating drum compared with the sham group, and T-006 treatment prolonged the holding time compared with the 6-OHDA group. These results suggest that T-006 can attenuate 6-OHDA-induced motor disorder in PD mice.

After the behavioral tests, the animals were sacrificed and the concentrations of dopamine and its metabolites (DOPAC, HVA) in the lesioned striatum were analyzed. As shown in Figure 5(d), we observed that injection of 6OHDA induced a significant decrease in the levels of dopamine, DOPAC, and HVA compared to the sham group. Interestingly, T-006 treatment significantly increased the concentrations of dopamine, DOPAC, and HVA compared with those of the 6-OHDA-lesioned mice.

We next investigated whether T-006 prevents 6-OHDAinduced degeneration of dopaminergic neurons in the $\mathrm{SNpc}$ in vivo. Immunohistochemical staining demonstrated that mice receiving saline injection presented with no significant difference in the number of TH-positive dopaminergic neurons compared to control mice (Figures 5(e) and 5(f)). The injection of 6-OHDA resulted in a marked loss of $\mathrm{TH}$ positive dopaminergic neurons in the lesioned side of the SNpc. By contrast, T-006 treatment conferred significant protection against 6-OHDA-induced death of dopaminergic neurons (Figures 5(e) and 5(f)).

Furthermore, we measured the density of TH-positive dopaminergic fibers in the striatum. 6-OHDA significantly decreased striatal dopaminergic fiber density compared with saline microinjection (Figures $2(\mathrm{~g})$ and $5(\mathrm{~h})$ ). Treatment with T-006 significantly attenuated the reduction of dopaminergic fiber density in the striatum. Altogether, these results indicate that T-006 is neuroprotective against 6-OHDA-induced toxicity in mice.

3.6. T-006 Treatment Activates PKA/Akt/GSK-3 $\beta$ Signaling in Brain Tissues. Based on the above outcome, we further investigated whether T-006 could activate PKA/Akt/GSK-3 $\beta$ signaling in vivo. C57BL/6 mice were administered with T-006 via oral gavage at different times. The phosphorylated and total protein levels of PKA, Akt, and GSK-3 $\beta$ were analyzed by Western blot. As shown in Figure 6, we found increased phosphorylation of PKA in brain tissues after T-006 treatment for $30 \mathrm{~min}$ compared with control mice. Similar variations in the phosphorylation of Akt were observed following T-006 treatment for $2 \mathrm{~h}$. The downstream target of Akt and GSK$3 \beta$ was also activated $4 \mathrm{~h}$ after injection of T-006. These results were consistent with those obtained following T-006 treatment of PC12 cells, suggesting that the neuroprotection conferred by T-006 may be, at least in part, mediated by the activation of the PKA/Akt/GSK-3 $\beta$ signaling pathway. 


\section{Discussion}

We have previously reported that the TMP derivative, T-006, exhibited a wide range of neuroprotective effects in $\mathrm{AD}$ models $[19,20]$. In the present study, we showed for the first time that T-006 also protects against 6-OHDA-induced neurotoxicity in PD models. We further studied the molecular mechanisms underlying the protective effects of T-006 in rat pheochromocytoma (PC12) cells and $\mathrm{C} 57 \mathrm{BL} / 6$ mice. We showed that T-006 prevented 6-OHDA-mediated depolarization of the mitochondrial membrane potential $(\Delta \psi \mathrm{m})$ and restored the energy metabolism and mitochondrial biogenesis in 6-OHDA-injured PC12 cells. Furthermore, T-006 significantly attenuated the loss of TH-positive neurons in the $\mathrm{SNpc}$, increased the concentration of dopamine and its metabolites (DOPAC, HVA) in the striatum, and restored motor functions in unilateral 6-OHDA-lesioned mice. The neuroprotective effects of T-006 are mediated, at least in part, by the activation of both the PKA/Akt/GSK-3 $\beta$ and CREB/PGC- $1 \alpha /$ NRF-1/TFAM pathways.

Mitochondria play a central role in the regulation of the cell apoptotic pathway, and a decrease in the mitochondrial membrane potential $(\Delta \psi \mathrm{m})$ has been shown to follow cell apoptosis. 6-OHDA has been widely used to generate both in vivo and in vitro models of PD [42], and the toxicity of 6 -OHDA was associated with the collapse in $\Delta \psi \mathrm{m}$. Here, we showed that pretreatment of PC12 cells with T-006 could attenuate 6-OHDA-induced neurotoxicity (Figure 1). Furthermore, it was observed that T-006 prevented 6-OHDAinduced $\Delta \psi \mathrm{m}$ loss, suggesting that T-006 may produce neuroprotective effects via the preservation of mitochondrial membrane integrity.

It is known that brain energy supply almost requires the oxidation of glucose through glycolysis, the TCA cycle, and oxidative phosphorylation [43, 44]. Accumulating evidence indicates that impaired energy metabolism plays a prominent role in the pathogenesis of PD [45], and the toxicity of 6OHDA is associated with the impairment of cellular energy metabolism through the collapse of glycolytic activity and inhibition of mitochondrial complexes I and IV. Here, we showed that T-006 could directly inhibit the decrease in glycolysis rate and mitochondrial respiratory capacity caused by 6-OHDA (Figure 3). We also observed that T-006 accelerated the TCA cycle, as evidenced by an increase in the important intermediate products including citrate, succinate, and oxaloacetate (Figure 3). Akt activation can increase the rate of glycolysis by promoting the translocation of glucose transporters (Glut1 and Glut4) to the cell surface and enhancing the activity of glycolytic enzymes (hexokinase and phosphofructokinase) $[46,47]$. Inhibition of GSK-3 $\beta$ (phosphorylated) by Akt resulted in the enzymatic activation of pyruvate dehydrogenase (PDH), which catalyzes the conversion of pyruvate into acetyl coenzyme A, thereby promoting the TCA cycle [47]. Moreover, Akt phosphorylates subunits $\alpha$ and $\beta$ of ATP synthase, resulting in an increase in mitochondrial respiration $[39,48]$. We further investigated the molecular mechanism by which T-006 promotes energy metabolism and showed that T-006 could activate Akt, which inhibited the activity of GSK- $3 \beta$ by increasing phosphoryla- tion at site Ser9 (Figure 4). Furthermore, adding T-006 rapidly increased p-PKA levels, while PKA inhibitor H-89 prevented the effects of T-006 on the phosphorylation of Akt and GSK-3 $\beta$ (Figure 4), suggesting that T-006 induced GSK- $3 \beta$ inactivation via PKA signaling cascade. More interestingly, we showed that T-006 significantly activated PKA/Akt/GSK-3 $\beta$ signaling in a mouse brain (Figure 6). These data indicate that T-006-promoted energy metabolism may involve these signaling pathways.

It is generally accepted that 6-OHDA-induced neurotoxicity is mediated by mitochondrial dysfunction [12, 49-51], and mitochondrial dysfunction is a major contributor to the pathogenesis of PD [52-54]. Promotion of mitochondrial biogenesis could restore mitochondrial function and protect against mitochondrial insults in PD [17, 55, 56]. Transcriptional coactivator PGC- $1 \alpha$ is a powerful regulator of mitochondrial biogenesis and cellular energy metabolism [17], and it has previously been reported that overexpression of PGC- $1 \alpha$ protects against neurodegenerative disease $[17,57]$. Our mechanistic studies demonstrate that T-006 could promote mitochondrial biogenesis (Figure 2) through upregulating the expression of PGC- $1 \alpha$, NRF1, and TFAM (Figure 5). In order to investigate the mechanisms underlying T-006-mediated promotion of mitochondrial biogenesis, we first analyzed the phosphorylation status of CREB, a transcription factor inducing PGC- $1 \alpha$ expression. We showed that T-006 significantly activated CREB within a short timeframe (i.e., within $15 \mathrm{~min}$ ) (Figure 2). Furthermore, the expression of $P G C-1 \alpha$ and its downstream gene is under the control of the PKA-CREB pathway (Figure 5). Our results were in agreement with other studies showing that PKA is an upstream factor regulating CREB [58]. We showed that T-006 increases phospho-CREB which could be completely abolished by PKA inhibitor H-89 (Figure 5). What is more, pretreatment with H-89 blocked mitochondrial biogenesis and attenuated the neuroprotection exerted by T-006 (Figure 5). These data demonstrate that T-006 protects PC12 cells against 6-OHDA-induced neurotoxicity through enhancing mitochondrial biogenesis via the PKA/CREB/PGC- $1 \alpha$ pathway.

It is well known that 6-OHDA induces loss of dopaminergic neurons in the SNpc through striatal dopamine depletion, which results in motor dysfunctions [42]. In the present study, we used this model for in vivo studies and injury was verified by an apomorphine-induced contralateral rotation test (Figure 6). We showed that treatment with T-006 significantly abolished the apomorphine-induced ipsilateral rotations resulting from 6-OHDA lesions (Figure 2). Moreover, 6-OHDA-lesioned mice exhibited significant impairment in motor function and spent less time on the rotating drum compared to the sham group; on the other hand, T-006 improved motor function after 2 weeks of treatment (Figure 2). Behavior change is probably associated with variations in the concentrations of dopamine and its metabolites [59]. We also show that 6-OHDA injection led to a major decrease in the content of dopamine and its metabolites (DOPAC, HVA) within the striatum, consistent with previous studies [59]. However, we find that T-006 can significantly increase the content of dopamine and its metabolites 
compared with lesioned mice (Figure 2). The decline in dopamine levels in PD is believed to arise from the severe loss of nigrostriatal dopaminergic neurons. It has been reported that 6-OHDA oxidizes and inhibits $\mathrm{TH}$, which is the ratelimiting enzyme in dopamine synthesis and is widely used as a marker for dopaminergic neurons [60]. More interestingly, we show that administration of T-006 can rescue the loss of TH-positive neurons on the lesioned side of the SNpc and attenuates the reduction of dopaminergic fiber density in the striatum (Figure 2).

\section{Conclusion}

The present study provided evidence that T-006 could significantly protect against 6-OHDA-induced neurotoxicity in PD models by promoting energy metabolism and mitochondrial biogenesis. The underlying molecular mechanism is, in part, mediated by the PKA/Akt/GSK-3 $\beta$ and PKA/CREB/PGC- $1 \alpha / \mathrm{NRF}-1 / \mathrm{TFAM}$ signaling pathways. Our results suggest that T-006 could be a promising candidate for the prevention and treatment of PD.

$\begin{array}{ll}\text { Abbreviations } \\ \text { 6-OHDA: } & \text { 6-Hydroxydopamine } \\ \text { DOPAC: } & \text { 3,4-Dihydroxyphenylacetic acid } \\ \text { ETC: } & \text { Electron transport chain } \\ \text { HVA: } & \text { Homovanillic acid } \\ \text { LDH: } & \text { Lactate dehydrogenase } \\ \text { OXPHOS: } & \text { Oxidative phosphorylation } \\ \text { PD: } & \text { Parkinson's disease } \\ \text { PMSF: } & \text { Phenylmethanesulfonyl fluoride } \\ \text { SNpc: } & \text { Substantia nigra pars compacta } \\ \text { TMP: } & \text { Tetramethylpyrazine } \\ \text { TH: } & \text { Tyrosine hydroxylase. }\end{array}$

\section{Data Availability}

The data used to support the findings of this study are available from the corresponding authors upon request.

\section{Conflicts of Interest}

The authors declare no actual or potential conflicts of interest.

\section{Authors' Contributions}

Hefeng Zhou, Min Shao, and Xuanjun Yang contributed equally to this study. Hefeng Zhou, Xuanjun Yang, Zaijun Zhang, and Simon Ming-Yuen Lee conceived and designed the experiments. Hefeng Zhou, Xuanjun Yang, Min Shao, Chuwen Li, Guozhen Cui, and Cheng Gao performed the experiments. Lijun Di, Hanbing Zhong, and Yuqiang Wang contributed in technical assistance and suggestions. Hefeng Zhou and Xuanjun Yang analyzed the data. Hefeng Zhou wrote the paper. All authors read and approved the manuscript.

\section{Acknowledgments}

This study was supported by grants from the Science and Technology Development Fund (FDCT) of Macao SAR (Ref. No. 069/2015/A2, No. 134/2014/A3, and No. 062-2017-AIR); Research Committee, University of Macau (MYRG201500182-ICMS-QRCM, MYRG2015-00214-ICMS-QRCM, MYRG139(Y1-L4)-ICMS12-LMY, and MYRG2016-00129ICMS-QRCM) awarded to Simon M.Y. Lee; and the National Science Foundation of China (81872842) awarded to Zaijun Zhang.

\section{Supplementary Materials}

Supplementary Material 1: Figure R1: the 2-DG abated T-006 protective effects on 6-OHDA-induced cytotoxicity. Supplementary Material 2: histological and stereological evaluation of TH-positive neurons. (Supplementary Materials)

\section{References}

[1] B. J. Ryan, S. Hoek, E. A. Fon, and R. Wade-Martins, "Mitochondrial dysfunction and mitophagy in Parkinson's: from familial to sporadic disease," Trends in Biochemical Sciences, vol. 40, no. 4, pp. 200-210, 2015.

[2] Y. Li, J. Wu, X. Yu et al., "The protective role of brain CYP2J in Parkinson's disease models," Oxidative Medicine and Cellular Longevity, vol. 2018, Article ID 2917981, 12 pages, 2018.

[3] L. V. Kalia and A. E. Lang, "Parkinson's disease," Lancet, vol. 386, no. 9996, pp. 896-912, 2015.

[4] L. V. Kalia and A. E. Lang, "Parkinson disease in 2015: evolving basic, pathological and clinical concepts in PD," Nature Reviews Neurology, vol. 12, no. 2, pp. 65-66, 2016.

[5] K. J. Doorn, P. J. Lucassen, H. W. Boddeke et al., "Emerging roles of microglial activation and non-motor symptoms in Parkinson's disease," Progress in Neurobiology, vol. 98, no. 2, pp. 222-238, 2012.

[6] A. H. Schapira, "Mitochondrial diseases," The Lancet, vol. 379, no. 9828, pp. 1825-1834, 2012.

[7] A. Ghosh, T. Tyson, S. George et al., "Mitochondrial pyruvate carrier regulates autophagy, inflammation, and neurodegeneration in experimental models of Parkinson's disease," Science Translational Medicine, vol. 8, no. 368, article 368ra174, 2016.

[8] R. Di Maio, P. J. Barrett, E. K. Hoffman et al., " $\alpha$-Synuclein binds to TOM20 and inhibits mitochondrial protein import in Parkinson's disease," Science Translational Medicine, vol. 8, no. 342, article 342ra78, 2016.

[9] D. Athauda and T. Foltynie, "Insulin resistance and Parkinson's disease: a new target for disease modification?," Progress in Neurobiology, vol. 145-146, pp. 98-120, 2016.

[10] R. Requejo-Aguilar, I. Lopez-Fabuel, E. Fernandez, L. M. Martins, A. Almeida, and J. P. Bolanos, "PINK1 deficiency sustains cell proliferation by reprogramming glucose metabolism through HIF1," Nature Communications, vol. 5, article 4514, 2014.

[11] H. Jiang, N. Song, H. Xu, S. Zhang, J. Wang, and J. Xie, "Up-regulation of divalent metal transporter 1 in 6hydroxydopamine intoxication is IRE/IRP dependent," Cell Research, vol. 20, no. 3, pp. 345-356, 2010.

[12] K. Hanrott, L. Gudmunsen, M. J. O'Neill, and S. Wonnacott, "6-Hydroxydopamine-induced apoptosis is mediated via 
extracellular auto-oxidation and caspase 3-dependent activation of protein kinase C $\delta$," Journal of Biological Chemistry, vol. 281, no. 9, pp. 5373-5382, 2006.

[13] E. M. Kerr, E. Gaude, F. K. Turrell, C. Frezza, and C. P. Martins, "Mutant Kras copy number defines metabolic reprogramming and therapeutic susceptibilities," Nature, vol. 531, no. 7592, pp. 110-113, 2016.

[14] I. Aviles-Olmos, P. Limousin, A. Lees, and T. Foltynie, "Parkinson's disease, insulin resistance and novel agents of neuroprotection," Brain, vol. 136, no. 2, pp. 374-384, 2013.

[15] H. C. Gao, H. Zhu, C. Y. Song et al., "Metabolic changes detected by ex vivo high resolution $1 \mathrm{H}$ NMR spectroscopy in the striatum of 6-OHDA-induced Parkinson's rat," Molecular Neurobiology, vol. 47, no. 1, pp. 123-130, 2013.

[16] C. T. Hong, K. Y. Chau, and A. H. Schapira, "Meclizineinduced enhanced glycolysis is neuroprotective in Parkinson disease cell models," Scientific Reports, vol. 6, no. 1, article 25344, 2016.

[17] K. Hasegawa, T. Yasuda, C. Shiraishi et al., "Promotion of mitochondrial biogenesis by necdin protects neurons against mitochondrial insults," Nature Communications, vol. 7, no. 1, article 10943, 2016.

[18] B. Zheng, Z. Liao, J. J. Locascio et al., " $P G C-1 \alpha$, a potential therapeutic target for early intervention in Parkinson's disease," Science Translational Medicine, vol. 2, no. 52, article 52ra73, 2010.

[19] D. Xu, H. Chen, S. Mak et al., "Neuroprotection against glutamate-induced excitotoxicity and induction of neurite outgrowth by T-006, a novel multifunctional derivative of tetramethylpyrazine in neuronal cell models," Neurochemistry International, vol. 99, pp. 194-205, 2016.

[20] H. Y. Chen, D. P. Xu, G. L. Tan et al., "A potent multifunctional neuroprotective derivative of tetramethylpyrazine," Journal of Molecular Neuroscience, vol. 56, no. 4, pp. 977-987, 2015.

[21] C. Zhang, C. Li, S. Chen et al., "Berberine protects against 6OHDA-induced neurotoxicity in PC12 cells and zebrafish through hormetic mechanisms involving PI3K/AKT/Bcl-2 and Nrf2/HO-1 pathways," Redox Biology, vol. 11, no. 1, pp. 1-11, 2017.

[22] C. M. Chong, D. Ma, C. Zhao et al., "Discovery of a novel neuroprotectant, BHDPC, that protects against MPP+/MPTPinduced neuronal death in multiple experimental models," Free Radical Biology \& Medicine, vol. 89, pp. 1057-1066, 2015.

[23] X. Yang, Y. Xu, T. Wang et al., "Inhibition of cancer migration and invasion by knocking down delta-5-desaturase in COX-2 overexpressed cancer cells," Redox Biology, vol. 11, pp. 653662, 2017.

[24] A. M. Zhang, R. Bi, Q. X. Hu, Y. Fan, Q. Zhang, and Y. G. Yao, "The OPA1 gene mutations are frequent in Han Chinese patients with suspected optic neuropathy," Molecular Neurobiology, vol. 54, no. 3, pp. 1622-1630, 2016.

[25] A. Janzer, N. J. German, K. N. Gonzalez-Herrera, J. M. Asara, M. C. Haigis, and K. Struhl, "Metformin and phenformin deplete tricarboxylic acid cycle and glycolytic intermediates during cell transformation and NTPs in cancer stem cells," Proceedings of the National Academy of Sciences of the United States of America, vol. 111, no. 29, pp. 1057410579, 2014.

[26] L. Tatenhorst, L. Tönges, K.-A. Saal et al., "Rho kinase inhibition by fasudil in the striatal 6-hydroxydopamine lesion mouse model of Parkinson disease," Journal of Neuropathology and Experimental Neurology, vol. 73, no. 8, pp. 770-779, 2014.

[27] L. Wang, J. Y. Chan, X. Zhou et al., "A novel agent enhances the chemotherapeutic efficacy of doxorubicin in MCF-7 breast cancer cells," Frontiers in Pharmacology, vol. 7, p. 249, 2016.

[28] H. S. Choi, H. J. Kim, J. H. Oh et al., "Therapeutic potentials of human adipose-derived stem cells on the mouse model of Parkinson's disease," Neurobiology of Aging, vol. 36, no. 10, pp. 2885-2892, 2015.

[29] F. Morroni, A. Tarozzi, G. Sita et al., "Neuroprotective effect of sulforaphane in 6-hydroxydopamine-lesioned mouse model of Parkinson's disease," NeuroToxicology, vol. 36, pp. 63-71, 2013.

[30] E. A. Mazzio, R. R. Reams, and K. F. Soliman, "The role of oxidative stress, impaired glycolysis and mitochondrial respiratory redox failure in the cytotoxic effects of 6hydroxydopamine in vitro," Brain Research, vol. 1004, no. 1-2, pp. 29-44, 2004.

[31] B. P. Dranka, J. Zielonka, A. G. Kanthasamy, and B. Kalyanaraman, "Alterations in bioenergetic function induced by Parkinson's disease mimetic compounds: lack of correlation with superoxide generation," Journal of Neurochemistry, vol. 122, no. 5, pp. 941-951, 2012.

[32] Y. Y. Glinka and M. B. Youdim, "Inhibition of mitochondrial complexes I and IV by 6-hydroxydopamine," European Journal of Pharmacology: Environmental Toxicology and Pharmacology, vol. 292, no. 3-4, pp. 329-332, 1995.

[33] Y. Glinka, M. Gassen, and M. B. Youdim, "Mechanism of 6hydroxydopamine neurotoxicity," Journal of Neural Transmission Supplementum, vol. 50, pp. 55-66, 1997.

[34] A. P. Bhatt, S. R. Jacobs, A. J. Freemerman et al., "Dysregulation of fatty acid synthesis and glycolysis in non-Hodgkin lymphoma," Proceedings of the National Academy of Sciences of the United States of America, vol. 109, no. 29, pp. 11818-11823, 2012.

[35] A. Hagiwara, M. Cornu, N. Cybulski et al., "Hepatic mTORC2 activates glycolysis and lipogenesis through Akt, glucokinase, and SREBP1c," Cell Metabolism, vol. 15, no. 5, pp. 725-738, 2012.

[36] H.L. Wieman, J.A. Wofford, and J.C. Rathmell, "Cytokine stimulation promotes glucose uptake via phosphatidylinositol-3 kinase/Akt regulation of Glut1 activity and trafficking," Molecular Biology of the Cell, vol. 18, no. 4, pp. 1437-1446, 2007.

[37] R. L. Elstrom, D. E. Bauer, M. Buzzai et al., "Akt stimulates aerobic glycolysis in cancer cells,” Cancer Research, vol. 64, no. 11, pp. 3892-3899, 2004.

[38] V. G. Antico Arciuch, S. Galli, M. C. Franco et al., "Akt1 intramitochondrial cycling is a crucial step in the redox modulation of cell cycle progression," PLoS One, vol. 4, no. 10, article e7523, 2009.

[39] C. Li, Y. Li, L. He et al., "PI3K/AKT signaling regulates bioenergetics in immortalized hepatocytes," Free Radical Biology \& Medicine, vol. 60, pp. 29-40, 2013.

[40] N. Filippa, C. L. Sable, C. Filloux, B. Hemmings, and E. Van Obberghen, "Mechanism of protein kinase $\mathrm{B}$ activation by cyclic AMP-dependent protein kinase," Molecular and Cellular Biology, vol. 19, no. 7, pp. 4989-5000, 1999.

[41] M. E. Hunzicker-Dunn, B. Lopez-Biladeau, N. C. Law, S. E. Fiedler, D. W. Carr, and E. T. Maizels, "PKA and GAB2 play central roles in the FSH signaling pathway to PI3K and AKT in ovarian granulosa cells," Proceedings of the National 
Academy of Sciences of the United States of America, vol. 109, no. 44, pp. E2979-E2988, 2012.

[42] P. Lindholm, M. H. Voutilainen, J. Laurén et al., "Novel neurotrophic factor CDNF protects and rescues midbrain dopamine neurons in vivo," Nature, vol. 448, no. 7149, pp. 73-77, 2007.

[43] L. Pellerin and P. J. Magistretti, "Let there be (NADH) light," Science, vol. 305, no. 5680, pp. 50-52, 2004.

[44] I. Georgakoudi and K. P. Quinn, "Optical imaging using endogenous contrast to assess metabolic state," Annual Review of Biomedical Engineering, vol. 14, pp. 351-367, 2012.

[45] L. Dunn, G. F. Allen, A. Mamais et al., "Dysregulation of glucose metabolism is an early event in sporadic Parkinson's disease," Neurobiology of Aging, vol. 35, no. 5, pp. 1111-1115, 2014.

[46] B. D. Manning and L. C. Cantley, "AKT/PKB signaling: navigating downstream," Cell, vol. 129, no. 7, pp. 1261-1274, 2007.

[47] B. Everts, E. Amiel, S. C. Huang et al., "TLR-driven early glycolytic reprogramming via the kinases TBK1-IKKe supports the anabolic demands of dendritic cell activation," Nature Immunology, vol. 15, no. 4, pp. 323-332, 2014.

[48] M. Capasso, M. K. Bhamrah, T. Henley et al., "HVCN1 modulates BCR signal strength via regulation of BCR-dependent generation of reactive oxygen species," Nature Immunology, vol. 11, no. 3, pp. 265-272, 2010.

[49] Y. Saito, K. Nishio, Y. Ogawa et al., "Molecular mechanisms of 6-hydroxydopamine-induced cytotoxicity in PC12 cells: involvement of hydrogen peroxide-dependent and -independent action," Free Radical Biology \& Medicine, vol. 42, no. 5, pp. 675-685, 2007.

[50] H. Liu, P. Mao, J. Wang, T. Wang, and C. H. Xie, "Allicin protects PC12 cells against 6-OHDA-induced oxidative stress and mitochondrial dysfunction via regulating mitochondrial dynamics," Cellular Physiology and Biochemistry, vol. 36, no. 3, pp. 966-979, 2015.

[51] X. Lu, J. S. Kim-Han, S. Harmon, S. E. Sakiyama-Elbert, and K. L. O'Malley, "The Parkinsonian mimetic, 6-OHDA, impairs axonal transport in dopaminergic axons," Molecular Neurodegeneration, vol. 9, no. 1, p. 17, 2014.

[52] M. T. Lin and M. F. Beal, "Mitochondrial dysfunction and oxidative stress in neurodegenerative diseases," Nature, vol. 443, no. 7113, pp. 787-795, 2006.

[53] W. Wang, X. Wang, H. Fujioka et al., "Parkinson's diseaseassociated mutant VPS35 causes mitochondrial dysfunction by recycling DLP1 complexes," Nature Medicine, vol. 22, no. 1, pp. 54-63, 2016.

[54] A. Grünewald, K. A. Rygiel, P. D. Hepplewhite, C. M. Morris, M. Picard, and D. M. Turnbull, "Mitochondrial DNA depletion in respiratory chain-deficient Parkinson disease neurons," Annals of Neurology, vol. 79, no. 3, pp. 366-378, 2016.

[55] R. Tufi, S. Gandhi, I. P. de Castro et al., "Enhancing nucleotide metabolism protects against mitochondrial dysfunction and neurodegeneration in a PINK1 model of Parkinson's disease," Nature Cell Biology, vol. 16, no. 2, pp. 157-166, 2014.

[56] W. Qi, H. A. Keenan, Q. Li et al., "Pyruvate kinase M2 activation may protect against the progression of diabetic glomerular pathology and mitochondrial dysfunction," Nature Medicine, vol. 23, no. 6, pp. 753-762, 2017.

[57] Q. Ye, W. Huang, D. Li et al., "Overexpression of PGC- $1 \alpha$ influences mitochondrial signal transduction of dopaminergic neurons," Molecular Neurobiology, vol. 53, no. 6, pp. 37563770, 2016.
[58] G. Lee, C. N. Ramirez, H. Kim et al., "Large-scale screening using familial dysautonomia induced pluripotent stem cells identifies compounds that rescue IKBKAP expression," Nature Biotechnology, vol. 30, no. 12, pp. 1244-1248, 2012.

[59] A. Kumar, D. Ganini, and R. P. Mason, "Role of cytochrome $c$ in $\alpha$-synuclein radical formation: implications of $\alpha$-synuclein in neuronal death in Maneb- and paraquat-induced model of Parkinson's disease," Molecular Neurodegeneration, vol. 11, no. 1, p. 70, 2016.

[60] S. Y. Won, M. H. Park, S. T. You et al., "Nigral dopaminergic PAK4 prevents neurodegeneration in rat models of Parkinson's disease," Science Translational Medicine, vol. 8, no. 367, article 367ra170, 2016. 


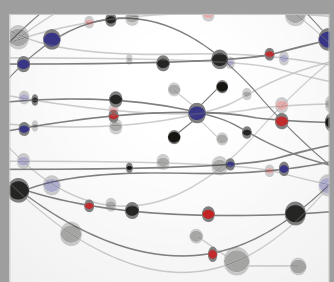

The Scientific World Journal
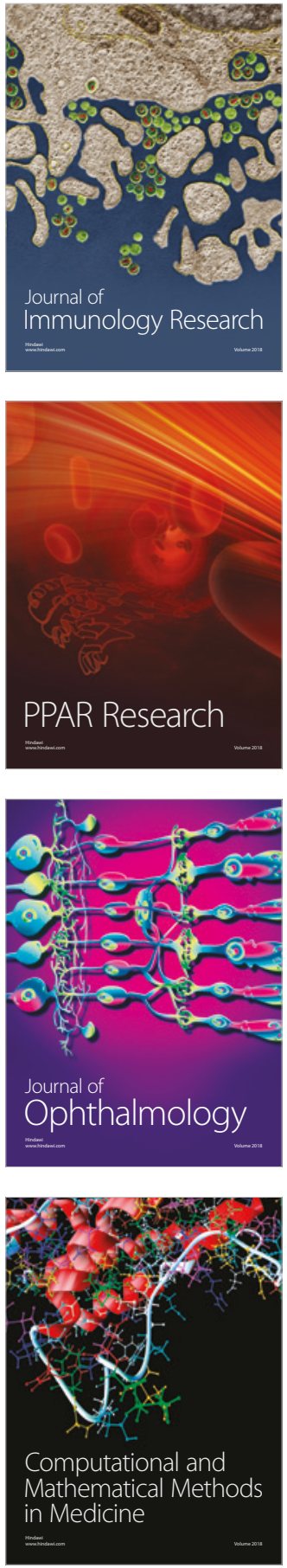

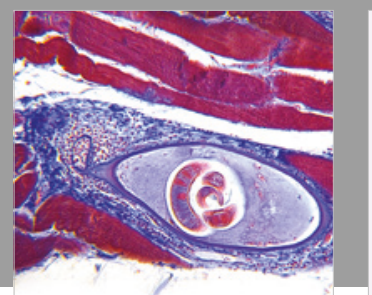

Gastroenterology Research and Practice

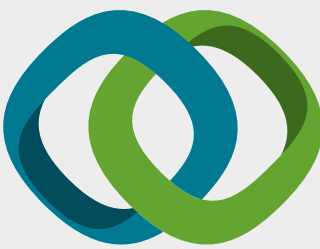

\section{Hindawi}

Submit your manuscripts at

www.hindawi.com
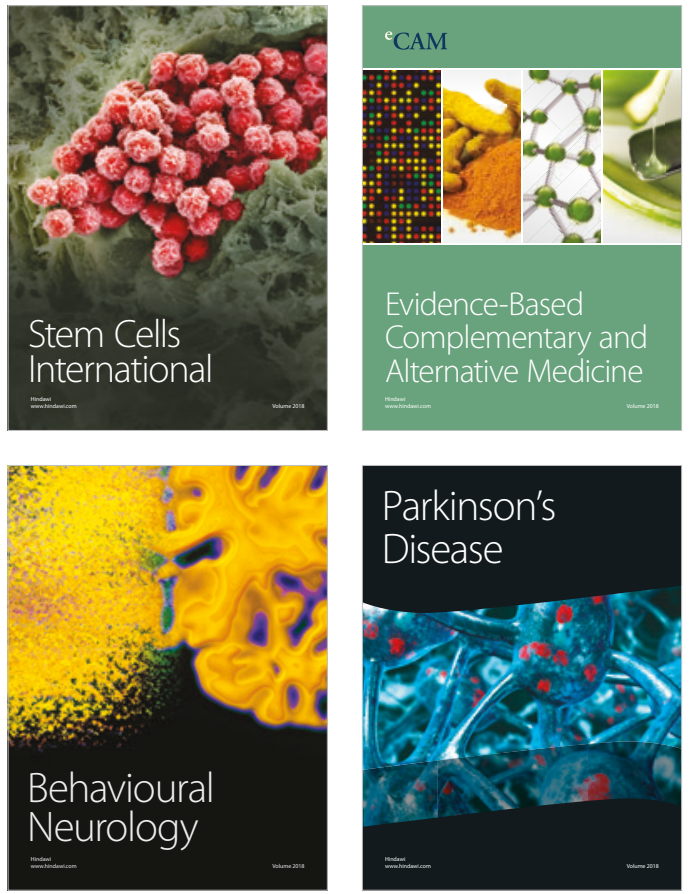

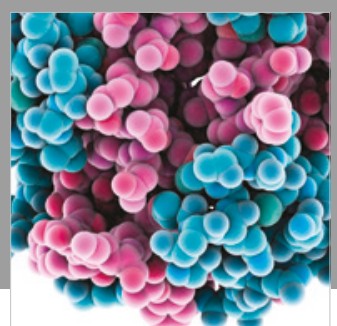

ournal of

Diabetes Research

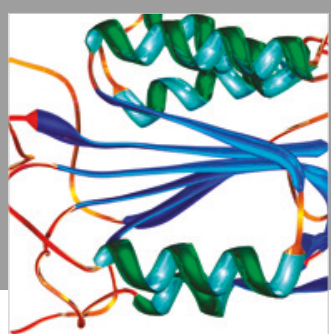

Disease Markers
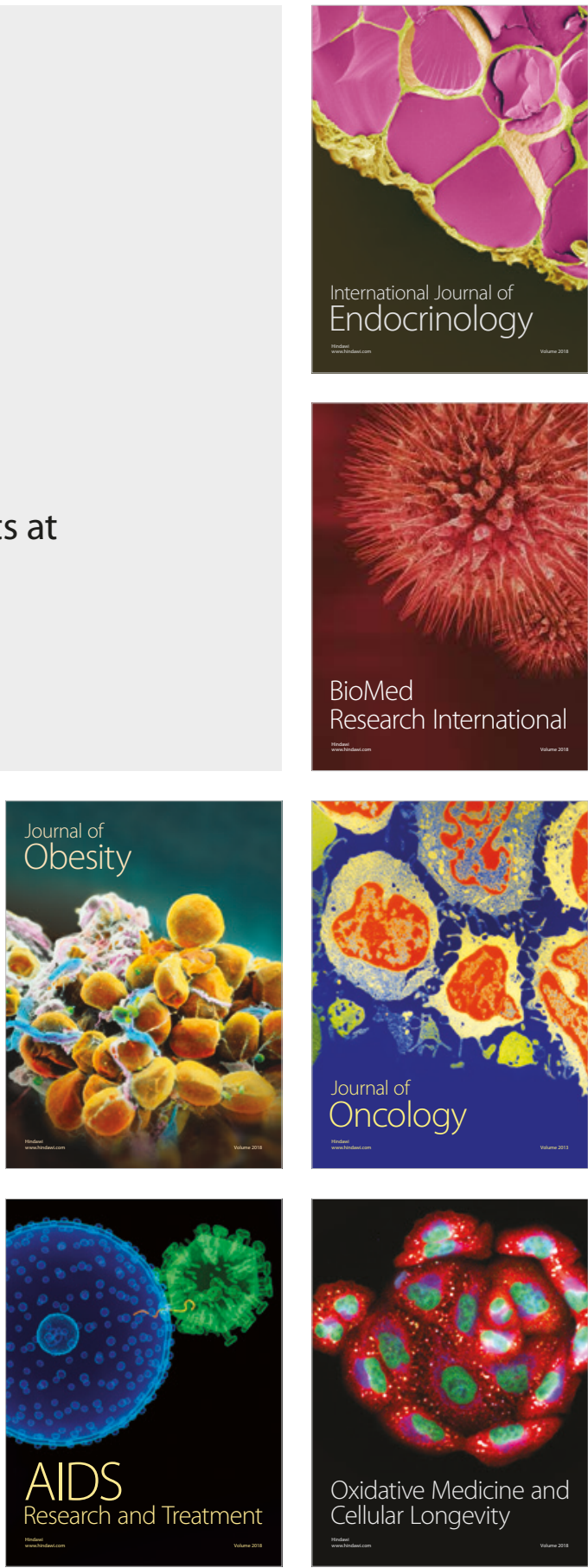